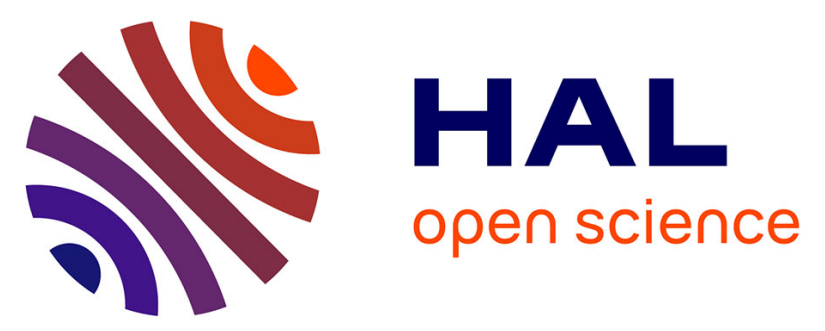

\title{
Task constraints act at the level of synergies and at the level of end-effector kinematics in manual reaching and manual lateral interception.
}

Inge Tuitert, Laura Golenia, Egbert Otten, Reinoud J Bootsma, Raoul M Bongers

\section{To cite this version:}

Inge Tuitert, Laura Golenia, Egbert Otten, Reinoud J Bootsma, Raoul M Bongers. Task constraints act at the level of synergies and at the level of end-effector kinematics in manual reaching and manual lateral interception.. Journal of Experimental Psychology: Human Perception and Performance, 2020, 46 (12), pp.1511-1526. 10.1037/xhp0000861 . hal-03091451

\section{HAL Id: hal-03091451 https://hal.science/hal-03091451}

Submitted on 31 Dec 2020

HAL is a multi-disciplinary open access archive for the deposit and dissemination of scientific research documents, whether they are published or not. The documents may come from teaching and research institutions in France or abroad, or from public or private research centers.
L'archive ouverte pluridisciplinaire HAL, est destinée au dépôt et à la diffusion de documents scientifiques de niveau recherche, publiés ou non, émanant des établissements d'enseignement et de recherche français ou étrangers, des laboratoires publics ou privés. 


\section{Journal of Experimental Psychology: Human Perception and Performance Task constraints act at the level of synergies and at the level of end-effector kinematics in manual reaching and manual lateral interception --Manuscript Draft--}

\begin{tabular}{|c|c|}
\hline Manuscript Number: & XHP-2019-1008R2 \\
\hline Full Title: & $\begin{array}{l}\text { Task constraints act at the level of synergies and at the level of end-effector kinematics } \\
\text { in manual reaching and manual lateral interception }\end{array}$ \\
\hline Abstract: & $\begin{array}{l}\text { To coordinate the redundant degrees of freedom (DOF) in the action system, synergies } \\
\text { are often proposed. Synergies organize DOF in temporary task-specific units emerging } \\
\text { from interactions amongst task, organism, and environmental constraints. We } \\
\text { examined whether task constraints affect synergies, end-effector kinematics, or both. } \\
\text { To this end, we compared synergies and end-effector kinematics when participants (N } \\
\text { = 15) performed discrete movements of identical amplitude in manual reaching } \\
\text { (stationary targets) and manual lateral interception (moving targets, with different } \\
\text { angles of approach). We found that time-velocity profiles were roughly symmetric in } \\
\text { reaching, whereas they had a longer decelerative tail and showed an angle-of- } \\
\text { approach effect in interception. Uncontrolled Manifold analyses showed that in all } \\
\text { conditions joint angle variability was primarily co-variation, indicating a synergistic } \\
\text { organization. The analysis on the clusters of joint angle configurations demonstrated } \\
\text { differences between reaching and interception synergies, whereas more similar } \\
\text { synergies were used within interception conditions. This implies that some task } \\
\text { constraints operate at the level of synergies while other task constraints only affect } \\
\text { end-effector kinematics. The results support a two-step process in the organization of } \\
\text { DOF, consisting of synergy formation and further constraining of synergies to produce } \\
\text { the actual movement, as proposed by Kay (1988). }\end{array}$ \\
\hline Article Type: & Research Report \\
\hline Keywords: & emergent synergies, reaching, interception, UCM analysis, end-effector kinematics \\
\hline Corresponding Author: & $\begin{array}{l}\text { Inge Tuitert } \\
\text { Universitair Medisch Centrum Groningen } \\
\text { NETHERLANDS }\end{array}$ \\
\hline Corresponding Author E-Mail: & i.tuitert@umcg.nl \\
\hline \multicolumn{2}{|l|}{$\begin{array}{l}\text { Corresponding Author Secondary } \\
\text { Information: }\end{array}$} \\
\hline Corresponding Author's Institution: & Universitair Medisch Centrum Groningen \\
\hline \multirow[t]{4}{*}{ Other Authors: } & Laura Golenia \\
\hline & Egbert Otten \\
\hline & Reinoud J Boostma \\
\hline & Raoul M. Bongers \\
\hline Author Comments: & $\begin{array}{l}\text { Dear Dr. Rob Gray, } \\
\text { We would like to submit the revised version of the paper "Task constraints act at the } \\
\text { level of synergies and at the level of end-effector kinematics in manual reaching and } \\
\text { manual lateral interception" to Journal of Experimental Psychology: Human } \\
\text { Performance and Perception. The manuscript number of the paper is: XHP-2019-1008. } \\
\text { The paper is co-authored by Inge Tuitert, Laura Golenia, Egbert Otten, Reinoud } \\
\text { Bootsma, and Raoul Bongers. We thank the reviewers for their comments, they really } \\
\text { helped us to improve the paper. The changes made are explained below. The } \\
\text { references to page and line numbers refer to the track changes document. We hope } \\
\text { that we succeeded in revising the paper in accordance with the comments of the } \\
\text { reviewers and that we have provided all relevant information appropriately. } \\
\text { Yours sincerely, } \\
\text { Inge Tuitert }\end{array}$ \\
\hline
\end{tabular}


Running head: TASK CONSTRAINT ACT AT SYNERGIES AND END-EFFECTOR KINEMATICS

Task constraints act at the level of synergies and at the level of end-effector kinematics in manual reaching and manual lateral interception

Tuitert I. ${ }^{1,2}$, Golenia L. ${ }^{2}$, Otten E. ${ }^{2}$, Bootsma R.J. ${ }^{1}$, and Bongers R.M. ${ }^{2}$

\footnotetext{
${ }^{1}$ Aix-Marseille Univ, CNRS, Institut des Sciences du Mouvement, Marseille, France

${ }^{2}$ University of Groningen, University Medical Center Groningen, Department of Human Movement Sciences, Groningen, the Netherlands
}

Correspondence concerning this article should be addressed to Inge Tuitert, Department of Human Movement Sciences, University Medical Center Groningen, PO box 196, 9700 AD Groningen.

Contact: i.tuitert@umcg.nl

Word count: 10890 


\section{TASK CONSTRAINT ACT AT SYNERGIES AND END-EFFECTOR KINEMATICS}

Abstract

To coordinate the redundant degrees of freedom (DOF) in the action system, synergies are often proposed. Synergies organize DOF in temporary task-specific units emerging from interactions amongst task, organism, and environmental constraints. We examined whether task constraints affect synergies, end-effector kinematics, or both. To this end, we compared synergies and end-effector kinematics when participants $(\mathrm{N}=15)$ performed discrete movements of identical amplitude in manual reaching (stationary targets) and manual lateral interception (moving targets, with different angles of approach). We found that time-velocity profiles were roughly symmetric in reaching, whereas they had a longer decelerative tail and showed an angle-of-approach effect in interception. Uncontrolled Manifold analyses showed that in all conditions joint angle variability was primarily co-variation, indicating a synergistic organization. The analysis on the clusters of joint angle configurations demonstrated differences between reaching and interception synergies, whereas more similar synergies were used within interception conditions. This implies that some task constraints operate at the level of synergies while other task constraints only affect end-effector kinematics. The results support a two-step process in the organization of DOF, consisting of synergy formation and further constraining of synergies to produce the actual movement, as proposed by Kay (1988).

Keywords: emergent synergies, reaching, interception, UCM analysis, end-effector kinematics 


\section{TASK CONSTRAINT ACT AT SYNERGIES AND END-EFFECTOR KINEMATICS}

\section{Significance statements}

A central question in the domain of motor control is how the redundant degrees of freedom (DOF) of the motor system (i.e., joints, muscles) are organized to perform goal-directed movements. It has been proposed that this organization occurs in two steps. First, DOF are organized in synergies, which are temporary task-specific units of DOF. These synergies are then further confined based on the specifics of the task, to produce the actual movement of a limb. To test this experimentally for the first time, we assessed synergies and movements of the index finger in discrete reaching and interception toward identical target (arrival) positions. We found that synergies differed between reaching and interception, but not among interception conditions, whereas the movements of the index finger differed across all these conditions. This supports the two-step process of organization of degrees of freedom in goal-directed actions. 


\section{TASK CONSTRAINT ACT AT SYNERGIES AND END-EFFECTOR KINEMATICS}

Task constraints act at the level of synergies and at the level of end-effector kinematics in manual reaching and manual lateral interception

Central in the domain of motor control research is the degrees of freedom (DOF) problem (Bernstein, 1967). The core of this problem is that the number of DOF (e.g., joint angles) used to perform a goal-directed activity usually exceeds the minimum necessary (cf. Greene, 1972; Turvey, Shaw, Reed, \& Mace, 1981; Turvey, 1990). To illustrate this, think of a planar arm with three joints keeping the end-effector at a target. Here, the three joint angles describe the joint configuration of the arm, while two coordinates describe the position of the end-effector in the plane. Because the number of DOF at joint level exceeds that at end-effector level, various joint angle configurations result in the same end-effector position. This so-called redundancy raises a key concern in studies of motor control questioning how the redundant DOF are coordinated to perform goal-directed actions (cf. Bernstein, 1967; Newell \& Vaillancourt, 2001). A coordinative principle suggested by Bernstein (1967) proposes how DOF are linked in a synergy. This suggestion inspired different approaches to gain a deeper understanding on the functioning of synergies over the last four decades (for pertinent overviews see Bruton \& O’Dwyer, 2018; Latash, Scholz, \& Schöner, 2007; Profeta \& Turvey, 2018; Turvey, 2007). In the current paper, we studied synergies from the dynamical systems perspective to movement coordination that understands synergies as flexible assemblies of DOF. Synergies are thus considered to be temporary organizations of DOF to perform a specific task or function. These synergies emerge from interactions amongst task, organism, and environmental constraints in a self-organizing manner (Kelso, 2009; Newell, 1986; Newell \& Vaillancourt, 2001; Profeta \& Turvey, 2018; Riley, Richardson, Shockley, \& Ramenzoni, 2011; Turvey, 1990, 2007). To understand the role of constraints in the formation of these synergies and their role in producing the resulting goal-directed behaviour, we examined whether different synergies emerged as a function of task constraints. 


\section{TASK CONSTRAINT ACT AT SYNERGIES AND END-EFFECTOR KINEMATICS}

To do this, we took the proposal of Kay (1988) as a starting point. Kay assumed that once the DOF are linked into a synergy (based on the interactions between task, organism, and environmental constraints), more specific constraints confine the synergy to produce the actual movement. Thus, constraints are proposed to act at two levels during the process of emerging behavior (Kay, 1988; Riley et al., 2011; Riley \& Turvey, 2002); constraints act (i) on the DOF to form an emergent synergy and, (ii) on the synergy to produce the actual behavior. To illustrate this, we consider the example of manual lateral interception, where the end-effector moves along a lateral displacement axis so as to intercept an approaching target that, over different trials, may follow different trajectories (different angles of approach) to the same interception position (e.g., Ledouit et al., 2013). The fact that a discrete movement has to be made could be one of the constraints that acts on the DOF, leading to the emergence of the synergy that moves the hand laterally. The target's angle of approach (and other constraints for that matter) may further constrain the synergy giving rise to the specifics of the movement to intercept the target (i.e., end-effector kinematics). Taking Kay's two-step framework as a starting point, we examined the effect of changes in task constraints on emergent synergies and end-effector kinematics to establish the tasks and conditions in which different synergies were used.

Two notions are of importance to gauge emergent synergies, and these notions require different methods of analyses. First, within an emergent synergy variation in one DOF is compensated for in other DOF so that task performance is stabilized (cf. Abbs \& Gracco, 1984; Kelso, Tuller, \& Fowler, 1984; Latash et al., 2007; Riley et al., 2011; Schettino, Adamovich, \& Tunik, 2017; Scholz \& Schöner, 1999; Schöner, 1995; Turvey, 2007). In terms of pointing at a target this means that a change in one joint angle is compensated by changes in one or more other joint angle(s) to preserve the position of the end-effector, that is, there is co-variation amongst joint angles. Therefore, we take the degree of co-variation to characterize the 


\section{TASK CONSTRAINT ACT AT SYNERGIES AND END-EFFECTOR KINEMATICS}

synergistic organization of DOF. Second, although there is a wide variety of configurations of DOF with which a given task can be performed, usually only a subset of these configurations is exploited. Think again about our example where an end-effector points at a target; here, there are many joint angle configurations that keep the end-effector at the target. Usually, not all of these options are employed: over repetitions of the same task, people typically only use a limited set of the available options of suitable joint angle configurations. Arguably, the total set of suitable configurations interacts with other constraints in the task, organism, and environment to form the synergy for that task. The subset or cluster of joint angle configurations actually used in positioning the end-effector, which has a certain location and size in joint space, emerges from these interactions and reflects the synergy (see Figure 1; cf. Profeta \& Turvey, 2018). Note that such a cluster of joint angles is different for different synergies. For instance, imagine reaching to a target on a table with the shoulder kept in place. This can be done by approaching a target from the left side or by approaching a target from the right side (see Figure 1), where these different approach sides might follow from instructions. The joint angle configuration will be different in those two actions and, arguably, the synergy is also different. Therefore, the cluster of joint angles used in a given task characterizes the synergy. In the current paper, the degree of co-variation in DOF as well as the cluster of DOF configurations used are examined to compare and characterize synergies.

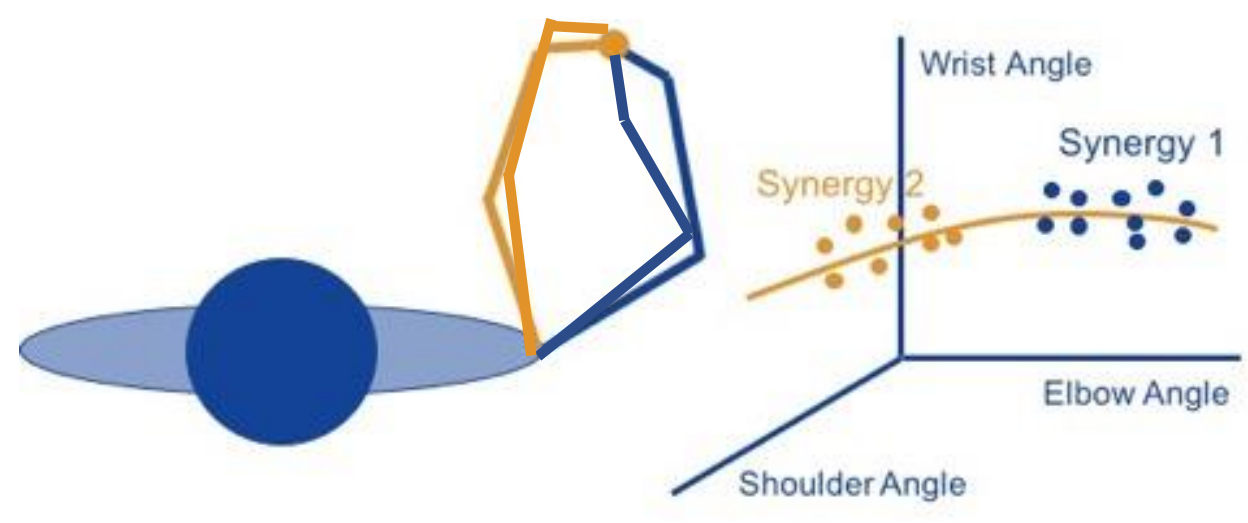




\section{TASK CONSTRAINT ACT AT SYNERGIES AND END-EFFECTOR KINEMATICS}

Figure 1. Schematic representation of two different synergies of a planar arm with three joints keeping the end-effector at a target having a certain location and size in joint space. The left part of the figure depicts from above a person with an arm, where the joint angle configurations in blue belong to synergy 1 and the joint angle configurations in orange belong to synergy 2 . The right part of the figure depicts the joint space, where the orange line represents the solution of joint angle combinations that keep the end-effector in that location. Each dot represents a joint angle configuration in a given 'trial'. Note that there is always some variation in actions over trials, hence the dots have some variation around the orange, solution, line. The different performances of the task (blue and orange postures) lead to clusters of joint angles that differ in location in joint space, see blue and orange dots. These different clouds joint angle configurations reflect different synergies, synergy 1 and synergy 2 , respectively.

A method to examine these aspects of synergistic organization of DOF is the Uncontrolled Manifold (UCM) analysis (Latash et al., 2007; Scholz \& Schöner, 1999; Schöner, 1995). This analysis partitions variability in DOF across repetitions of trials into two types. The first type of variability is variability within the set of DOF configurations that stabilizes a performance variable (i.e., co-variation), that is, variability in joint angles that does not affect end-effector position. The other type of variability in DOF leads to deviations of the performance variable, that is, joint angle variability moving the end-effector away from a certain position. The variability in DOF not affecting the end-effector position (i.e., co-variation) should be larger than the variability in DOF affecting the end-effector position to execute a given task. Therefore, we use the degree of co-variation as a measure to assess synergistic organization of DOF. In addition, a variation on an adapted version of the UCM method (Schöner, 2008; see also, Mattos, Latash, Park, Kuhl, \& Scholz, 2011) will be used in the current study to compare the location and size in joint space of the clusters of employed joint angle configurations 


\section{TASK CONSTRAINT ACT AT SYNERGIES AND END-EFFECTOR KINEMATICS}

between tasks and among conditions within one task. These clusters of joint angle configurations are taken to reflect the subset from the total set of suitable joint angle configurations employed by the synergies.

We applied these methods in two tasks involving the upper extremity where a set of task constraints related to timing and guidance towards the target were varied. The task constraints followed from the specifics of the setup, related to for instance target size and distance, and from instructions of how to perform the task. The two tasks were manual reaching and manual lateral interception, bringing the end-effector toward identical target (arrival) positions. In both tasks the hand sled along a lateral axis, creating frictions that acted as a task constraint. In the manual reaching task, participants were instructed to move the tip of the index-finger from a start position to a stationary target position, in the lateral direction, by sliding the end-effector along a lateral axis. Aside from the instruction to move as fast as possible while ending the movement in the target, no particular constraints on speed or movement time were imposed. In the lateral manual interception task, participants had to intercept a moving target that approached the participant in the horizontal plane by sliding the end-effector along the same lateral axis. The moving target imposed a timing constraint and guided the movement of the index-finger to intercept the target at the arrival position. To modulate the guidance of the movement of the index-finger, we varied the target's trajectory (approach angle) to the final arrival position (e.g., Ledouit, Casanova, Zaal, \& Bootsma, 2013; Michaels, Jacobs, \& Bongers, 2006; Montagne, Laurent, Durey, \& Bootsma, 1999; Peper, Bootsma, Mestre, \& Bakker, 1994).

What is known about the use of synergies and kinematics in these two tasks? The kinematics of the end-effector have been examined extensively, yet, separately for manual reaching (e.g., Flash \& Hogan, 1985; Gordon, Ghilardi, \& Ghez, 1994; Morasso, 1981) and manual lateral interception (e.g., Ledouit et al., 2013; Michaels et al., 2006; Montagne et al., 1999; Peper et al., 1994). In manual reaching without stringent precision constraints (as in a 


\section{TASK CONSTRAINT ACT AT SYNERGIES AND END-EFFECTOR KINEMATICS}

difficult Fitts' task), a main kinematic feature is a roughly symmetric bell-shaped velocity profile (Flash \& Hogan, 1985; Morasso, 1981) where the maximum velocity of the end-effector increases with target distance (Gordon et al., 1994). In general, the symmetry of the velocity profile is not affected by target distance (Gordon et al., 1994). In manual lateral interception, the velocity profile depends on characteristics of the trajectory of the target; for example, if the target motion duration is sufficiently long, the velocity profile demonstrates a longer deceleration tail (Ledouit et al., 2013). Moreover, the angle of approach of the target influences the velocity profile of the hand to the interception location in a systematic way (Ledouit et al., 2013; Michaels et al., 2006; Montagne et al., 1999; Peper et al., 1994). Based on these findings in the literature, we foresee differences in end-effector kinematics between tasks and amongst interception conditions. We expect that the velocity profiles of manual reaching will be roughly bell-shaped, whereas the velocity profiles in manual lateral interception are expected to have longer deceleration tails and portray an angle-of-approach effect.

The use of joint angle synergies has been studied in manual reaching; however, studies addressing this issue in interception are sparse. Using UCM analyses, it has been demonstrated that the joint angles of the arm stabilize the end-effector position (e.g., de Freitas, Scholz, \& Stehman, 2007; Golenia, Schoemaker, Otten, Tuitert, \& Bongers, 2018; Greve, Zijlstra, Hortobágyi, \& Bongers, 2013; Hansen, Grimme, Reimann, \& Schöner, 2015; Krüger, Eggert, \& Straube, 2013; Mattos et al., 2011; Tseng, Scholz, Schöner, \& Hotchkiss, 2003; Valk, Mouton, \& Bongers, 2016; Van Der Steen \& Bongers, 2011), implying that the joint angles are organized in synergies in reaching. Furthermore, the range of joint angle configurations employed by a synergy has been found to be larger in specific conditions. For instance, it is higher in more challenging conditions compared to a control condition (de Freitas et al., 2007), and during learning it is higher at the end of learning compared to at the start (Yang \& Scholz, 2005; Yang, Scholz, \& Latash, 2007), or in mid-childhood development it is higher than in 


\section{TASK CONSTRAINT ACT AT SYNERGIES AND END-EFFECTOR KINEMATICS}

adults (Golenia et al., 2018; Golenia, Schoemaker, Otten, Mouton, \& Bongers, 2018). In manual lateral interception, synergies have not been studied using UCM analysis. However, in other interception tasks the influence of varying task constraints on the linking of joints within a synergy has been addressed with analysis other than the UCM (Bockemühl, Troje, \& Dürr, 2010; Mazyn, Montagne, Savelsbergh, \& Lenoir, 2006). For example, in unrestrained onehanded catching of real objects with large variations in target arrival positions, the couplings amongst several joints have been quantified (Bockemühl et al., 2010) and couplings between pairs of joints have been shown to be stronger when the objects approached faster (Mazyn et al., 2006). Based on these previous findings we predict that synergies arise in both tasks used in the current study.

In summary, we asked whether different synergies are used when task constraints are varied to create different tasks and conditions in manual goal-directed reaching. We started from the assumption that task constraints act separately at the level of synergies and at the level of endeffector kinematics. Synergies were characterized by (i) the degree of co-variation amongst joint angles over repetitions of the same task and condition and (ii) the cluster of joint angle configurations that the synergy employed in a condition. Our analyses were structured in the following way. First, we tested whether the shape of the velocity profiles of the end-effector differed between manual reaching and manual interception and amongst interception conditions. Based on previous findings, we expected that the velocity profiles differ. Second, we investigated whether joint angles were organized in synergies in all manual reaching and manual lateral interception conditions, using the UCM analysis (Latash et al., 2007; Scholz \& Schöner, 1999; Schöner, 1995). Synergies have been revealed in manual reaching and we expect to also find synergies in manual lateral interception. Third, we assessed whether synergies differed between manual reaching and manual interception and amongst interception 


\section{TASK CONSTRAINT ACT AT SYNERGIES AND END-EFFECTOR KINEMATICS}

conditions. To do this we used an adapted version of the motor equivalent analysis proposed by Schöner (2008; see also, Mattos et al., 2011).

\section{Methods}

\section{Participants}

The sample size was estimated using G*Power (Version 3.1; Faul, Erdfelder, Buchner, \& Lang, 2009) based on the UCM analysis, where we hypothesized to find a larger $V_{\text {ucm }}$ than $V_{\text {ort. }}$ Former studies (Greve, Hortobagyi, \& Bongers, 2015; Golenia et al., 2018) showed strong effects for the difference between $V_{u c m}$ and $V_{\text {ort }}$. Note that we were also interested in effects on differences in clusters of joint angles. However, we had no appropriate data to compute a power analysis on these effects. We therefore took a relatively more conservative effect size $(f=0.4)$ and a correlation between measurements of 0.5 for sample size estimation. Fifteen participants would allow the detection of an effect at alpha $=0.05$ with a power of 0.8 for a repeatedmeasures analysis of variance with a two-level within-subject variable. This number is in line with the number of participants included in earlier studies detecting differences between conditions in a rod reaching experiment focusing on $\mathrm{V}_{\text {ucm }}$ and $\mathrm{V}_{\text {ort }}$ (e.g., Valk et al., 2016). Therefore, we aimed at including 15 of participants.

Fifteen right-handed adults ( 8 males and 7 females, mean age 25 years, standard deviation 9 years) participated in the experiment. All participants had normal or corrected-to-normal vision. The study was approved by the local Ethical Board of the Center for Human Movement Sciences of the University Medical Center Groningen, Groningen, The Netherlands. Written informed consent was obtained from all participants included in the study prior to the start of the experiment.

\section{Apparatus}

Participants were seated on a chair of which the backrest was extended with a plate. The trunk of the participants was gently strapped to this plate (Domkin, Laczko, Jaric, Johansson, 


\section{TASK CONSTRAINT ACT AT SYNERGIES AND END-EFFECTOR KINEMATICS}

\& Latash, 2002; Van Der Steen \& Bongers, 2011) to prevent movements of the trunk while permitting free rotations in the shoulder joints. The chair was placed at the long edge of a table in which a flat screen television (Panasonic TH-50PY70F; screen size 110.5 x $62 \mathrm{~cm}, 1920 \mathrm{x}$ 1080 m pixel resolution) was horizontally embedded. Participants rested their elbow on an arm rest at the start of each trial to standardize the starting posture as much as possible across trials.

3D positions of IREDs (infrared light-emitting diodes) attached to the body were measured at a sampling frequency of $100 \mathrm{~Hz}$ with two Optotrak 3020 system sensors using First Principles (Northern Digital, Waterloo, Canada). Six triangular rigid PVC plates, with each three IREDs (one in each corner of the triangle) were attached to the participant's sternum, the acromion, on the lateral side of the right upper arm below the insertion of the deltoid, proximal to the ulnar and radial styloids, to the dorsal surface of the hand (van Andel, Wolterbeek, Doorenbosch, Veeger, \& Harlaar, 2008), and to the index-finger (Van Der Steen \& Bongers, 2011), using skin-friendly tape. A small aluminum plate was taped under the index-finger to prevent flexionextension in the interphalangeal joints while allowing for flexion-extension and adductionabduction in the metacarpophalangeal joint. Following the procedure described by Van Andel et al. (2008), the positions of the six rigid bodies were linked to 19 local anatomical positions for each participant, using a standard pointer device. The fingertip position along the lateral axis of the television screen was also recorded using a draw-wire potentiometer (FDMK461000-P10, Altheris, the Netherlands). The potentiometer was placed at the left bottom of the table and its wire was attached to the fingertip with Velcro tape.

\section{Procedure}

The experiment commenced with attaching the rigid bodies to the dominant right arm and the trunk. Subsequently, the anatomical landmarks were recorded. After this procedure, the participant was strapped to the chair and the draw-wire was attached to the index-finger. In the two tasks of the experiment (reaching and interception) the starting location of the right 


\section{TASK CONSTRAINT ACT AT SYNERGIES AND END-EFFECTOR KINEMATICS}

fingertip was always aligned with the body midline at the near edge of the screen (see Figure 2). Participants were instructed to slide the index-finger along the edge of the screen during the movement towards the target in both tasks.

Reaching. A reaching trial began with the presentation of the start target and the goal target (both 1.5-cm diameter circles) at the near side of the screen. Goal targets could be located at distances of 20,30 or $40 \mathrm{~cm}$ to the right of the start target (see Figure 2), all on a lateral axis at the edge of the tv screen perpendicular to the sagittal plane. After the appearance of the targets, participants placed their index finger on the start target, with the elbow at the elbow rest, and initiated their movement at their own convenience. After movement initiation the elbow could be moved without any restrictions. The instruction for the reaching movements was to reach the goal target as fast and accurate as possible, while sliding the fingertip across the screen. No feedback about the performance was provided.

Interception. An interception trial started with the presentation of the start target at the near side of the screen and the goal target (2-cm diameter circle) at the far side. After remaining at its initial position for $1.5 \mathrm{~s}$, the goal target started to move across the screen towards its near side, arriving there after $1 \mathrm{~s}$ (orthogonal velocity $60 \mathrm{~cm} / \mathrm{s}$ ). The participant was instructed to initiate the interceptive action after the goal target had started to move (trials where participants moved within $150 \mathrm{~ms}$ after the goal target started moving were excluded to control for this). The instruction for the interceptive action was to intercept the goal target by moving the fingertip along the lateral axis reaching low velocity at the instant of interception. Goal target arrival positions on the interception axis were identical to those used in the reaching task (20, 30 , and $40 \mathrm{~cm}$ to the right of the start target). The goal target could arrive at these arrival positions with one of the three angles of approach $\left(-26.5^{\circ}, 0^{\circ}, 26.5^{\circ}\right.$; Outward, Straight, Inward, respectively; see Figure 2). Based on the fingertip position data provided by the draw-wire potentiometer, interception was defined as being within a $3-\mathrm{cm}$ range around the middle of the 


\section{TASK CONSTRAINT ACT AT SYNERGIES AND END-EFFECTOR KINEMATICS}

target arrival position, taken with respect to the middle of the index-finger. Participants were provided with this feedback immediately after each trial, which could either be "intercepted", "missed at left side", or "missed at right side", depending on performance.

\section{Design}

The reaching and interception tasks were performed in separate blocks, with task order randomized across participants. In the reaching task, participants performed 30 trials for each goal target position condition, presented in randomized order, resulting in a total of 90 reaching trials per participant. In the interception task, participants performed 30 trials under each combination of angle of approach and goal target arrival position conditions, presented in randomized order, resulting in a total of 270 interception trials per participant. The full experiment thus included 360 trials per participant.

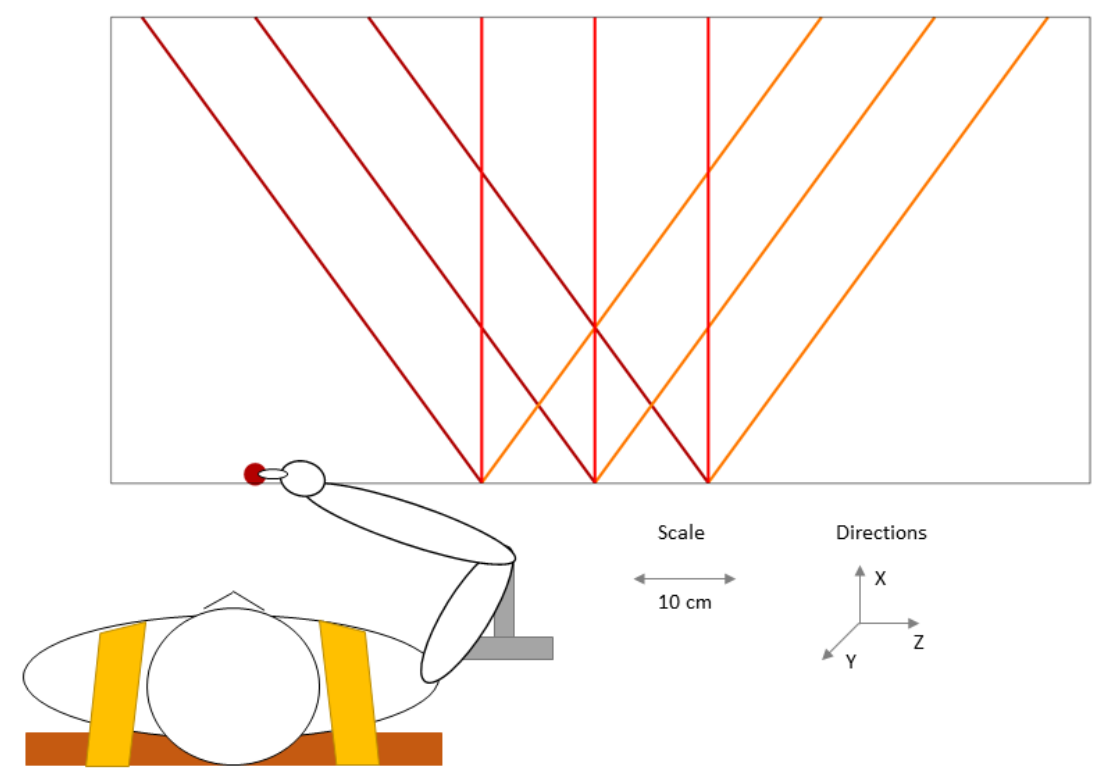

Figure 2. Bird's-eye view of the experimental set-up and target trajectories of the interception conditions. The large rectangle represents the screen. The dot is the start target, and the moving targets were intercepted along this lower lateral axis. The dark red (dark grey) lines are the $26.5^{\circ}$ (outward) angle of approach, the red (grey) lines are the $0^{\circ}$ (straight) angle of approach, 


\section{TASK CONSTRAINT ACT AT SYNERGIES AND END-EFFECTOR KINEMATICS}

and the orange (light grey) lines are the $26.5^{\circ}$ (inward) angle of approach of the moving targets. The elbow of the participant is on the grey stand and the fingertip of the participant is on the start target. The participant is strapped to the brown chair with the yellow bandage. The size of the participant is an approximation, implying that the scale shown in the figure applies to the table and does not apply to the participant.

\section{Data analysis}

The data were analyzed using customized programs written in Matlab (Mathworks, Natick, MA). The percentage of intercepted balls in the interception trials was calculated using the feedback that was given to the participants which was based on the draw-wire potentiometer data. Subsequently, missed interception trials were excluded from the analysis. After filtering the X-Y-Z time series of fingertip position with a cut-off frequency of $5 \mathrm{~Hz}$ (low-pass secondorder Butterworth filter ran through twice to negate the phase shift), the lateral (Z) velocity was computed using a three-point central difference method. For each trial, the start of the movement was determined by searching backward in time, starting from peak velocity. The first data point where the velocity fell below a threshold of $5 \mathrm{~cm} / \mathrm{s}$ was marked as the start of the movement. In the reaching trials, the end of the movement was marked as the first data point where the velocity fell below a threshold of $5 \mathrm{~cm} / \mathrm{s}$ when searching forward from peak velocity. In interception trials, the instant of interception (the time instant where the goal target passed the near end of the screen, $1 \mathrm{~s}$ after the target started moving) was marked as the end of the movement. Movement Time was defined as the time between the start and the end of the movement. The Variable Error of the end-effector was computed as the standard deviation of the difference between the end-effector lateral position at the end of the movement and the lateral position of the middle of the target (arrival) position. For the kinematic analysis, the uncontrolled manifold analysis and the analyses on the clusters of joint angles (see upcoming 


\section{TASK CONSTRAINT ACT AT SYNERGIES AND END-EFFECTOR KINEMATICS}

sections) each trial was time-normalized to 100 steps, between the start and the end of the movement. For analyses on the clusters of joint angles, each trial was also lateral distance normalized. Within a condition where time start and end point were detected, the most rightward start $\mathrm{Z}$ position and the most leftward end $\mathrm{Z}$ position were detected across trials. The data was spatial-normalized to 10 steps, between the detected start and end $\mathrm{Z}$ position. For clarity, not all results are presented time- and spatial-normalized. The spatial-normalized data of a representative participant is depicted in a figure to show the similarities in results.

Kinematics. To statistically test differences in kinematics, we analyzed Movement Time and Symmetry Index of Velocity, which was defined as the time needed to reach peak velocity divided by Movement Time. A Symmetry Index of Velocity of about 0.5 indicates a bell-shaped velocity pattern which is expected in reaching, whereas a Symmetry Index of Velocity smaller than 0.5 indicates a longer decelerative tail which is expected in interception.

Synergistic organization of joint angles: UCM analysis. Joint rotations were computed following International Society of Biomechanics (ISB) guidelines for the upper extremity (Wu et al., 2005): shoulder plane of elevation, shoulder elevation, shoulder inward-outward rotation, elbow flexion-extension, forearm pronation-supination, wrist flexion-extension, wrist abduction-adduction, index-finger flexion-extension, and index-finger abduction-adduction. The UCM method requires four steps (Latash et al., 2007). As first two steps, the elemental variables selected were the nine joint angles while the 3D position of the tip of the index finger was selected as the performance variable. Subsequently, small changes in the joint angles were related to small changes in the index-finger position by means of a linear model (third step) and were represented in a Jacobian matrix. Lastly, this matrix was used to partition the joint-angle variance across trials; $\mathrm{V}_{\mathrm{ucm}}$ is the variance within the null space of the Jacobian (i.e., variability in joint angles not affecting end-effector position) and $V_{\text {ort }}$ is the variance orthogonal to the null space (i.e., variability in joint angles affecting end-effector position). Each $U C M\left(V_{u c m}\right.$ and $\left.V_{\text {ort }}\right)$ 


\section{TASK CONSTRAINT ACT AT SYNERGIES AND END-EFFECTOR KINEMATICS}

component was normalized by its number of DOF, and computed for each condition and each target (arrival) location separately. For the equations used in these four computational steps, see Tuitert et al. (2017). If $\mathrm{V}_{\text {ucm }}$ is larger than $\mathrm{V}_{\text {ort }}$, there is mainly co-variation amongst joint angles. The relation between these two variables reflects the degree of co-variation. To correct for non-normal data distribution $\mathrm{V}_{\mathrm{ucm}}$ and $\mathrm{V}_{\text {ort }}$ were $\log$ transformed prior to the statistical analysis (Verrel, 2010).

Clusters of joint angle configurations. The goal of the current analyses was to establish whether different tasks and different conditions were performed with different synergies (see also Valk, Mouton, Otten, \& Bongers, 2019b). It is important to realize that the main variables in this analysis were the lengths of projection vectors, whereas in the UCM analysis the variances of these vectors were computed. The way we implemented these analyses required to use one condition as a base condition to which the cluster of joint angle configurations in other conditions were compared. The method works as follows. First, the UCM of the base condition is computed. Then the individual trials of the base condition are projected on the UCM of the base. The projection lengths in the null-space and in the orthogonal space are computed. For each space a confidence interval is computed of the projection lengths of the base trials. The next step is to project the individual trials of another condition on the UCM of the base condition. Again, the projection lengths are computed in the null-space and the orthogonal space. For both spaces, it is examined whether these projection lengths fall outside the confidence interval of the base condition. When these projections fall outside, we classify that as another synergy as the base condition. A more detailed explanation of the followed procedure can be found in the paragraph below.

We chose the Interception Straight (IS) condition as the base condition. This was done to be able to compare reaching with interception, while also making it possible to compare different interception conditions. Note, as a check we performed similar analyses with another 


\section{TASK CONSTRAINT ACT AT SYNERGIES AND END-EFFECTOR KINEMATICS}

interception condition as a baseline and this did not affect the findings. All the computations reported hereafter were performed separately for each (distance) condition of each participant. First, we selected the null-spaces and orthogonal spaces of the Jacobian of the base conditions calculated for the UCM analysis as the bases. Then, joint deviation vectors (JDVAs) were computed as the real difference between the average joint angle configuration of the base condition and the average joint angle configuration of each of the remaining conditions (Reaching (R), Interception Outward (IO), Interception Inward (II); see Mattos et al., 2011). These JDVAs were projected onto the null-space of the base Jacobian and onto its orthogonal complement, resulting in the measures PRA $\mathrm{ucm}_{\mathrm{uc}}, \mathrm{PIOA}_{\mathrm{ucm}}, \mathrm{PIIA}_{\mathrm{ucm}}, \mathrm{PRA}_{\text {ort}}, \mathrm{PIOA}_{\text {ort }}$ PIIA $\mathrm{A}_{\text {ort, }}$ respectively (P stands for projection, A stands for average). The length of the projection onto the base null-space represents an estimate of the change in the joint angle configurations as a result of the change in task constraints between the base and the projected condition, which did not affect the fingertip position $\left(\mathrm{P}_{\mathrm{ucm}}\right)$. The length of the projection onto the base orthogonalspace represents an estimate of that change that does influence the fingertip position $\left(\mathrm{P}_{\text {ort }}\right)$. Each projection component was normalized by the square root of its number of DOF (Mattos et al., 2011), 6 DOF for the null-space projection and 3 DOF for the orthogonal-space projection. These measures give an indication of the distance of the average joint angle configuration of a condition to the base condition, for both the null-space and the orthogonal space separately. Note this analyses takes into account co-variation between joint angles, because the joint angle configuration is assessed separately for null-space and orthogonal space. The motor equivalence analysis by Mattos et al. (2011) assesses whether $\mathrm{P}_{\mathrm{ucm}}$ is larger than $\mathrm{P}_{\text {ort }}$ to quantify motor equivalence. We aimed to quantify whether synergies in terms of employed joint angle configurations differed, therefore, we expanded their analysis from this point onward. To establish differences in synergies between two conditions we computed a benchmark for the base condition to which the other conditions were compared. To compute the benchmark for 


\section{TASK CONSTRAINT ACT AT SYNERGIES AND END-EFFECTOR KINEMATICS}

the base condition we computed the difference between the joint angle configurations of the individual base trials and the average joint angle configuration of the base. We projected these difference vectors on the null-space and orthogonal-space of the base condition. These projections can be represented as vectors that have a certain length. The lengths of the projections reflect the deviation from the mean for each separate trial (of the base condition) in the null-space and orthogonal-space for the base condition. Again, these lengths were normalized by the square root of their number of DOF, 6 DOF for the null-space projection and 3 DOF for the orthogonal-space projection. Using the lengths of these projections, we calculated the upper bound of the $95 \%$ confidence interval for the projections onto the null space and the orthogonal space, which was chosen to represent the boundaries of the base synergy. Note that we did not use the lower bound of the confidence interval, because it is very close to the origin of the null space, as the null space is computed using small changes in the mean joint angle configuration of the base condition. Hence, the lower bound does not inform about the boundary of the base condition. We refer to these upper bounds as the projection bases $\left(\mathrm{PB}_{\mathrm{ucm}}\right.$ and $\left.\mathrm{PB}_{\text {ort}}\right)$.

We commenced the analyses on the differences in clusters of joint angles for the different conditions by comparing PRA, PIOA, and PIIA to PB separately for each space (UCM and ORT) to establish differences between clusters of joint angle configurations (i.e., synergies). That is, we compared the PRA, PIOA, and PIIA to the boundary of the confidence interval of the PB, for each space separately (UCM and ORT).

In addition to the average JDVA analyses, we also assessed the separate trials of the R, IO, and II conditions, as a large variation across trials was expected. JDV vectors were computed as the real difference between the average joint angle configuration of the base condition and the joint angle configuration of an individual trial. The projection and normalization of the trials were computed as described before, resulting in a PR, PIO and PII per trial. Thereafter, we 


\section{TASK CONSTRAINT ACT AT SYNERGIES AND END-EFFECTOR KINEMATICS}

compared the PR, PIO, and PII to the boundary of the confidence interval of the PB, for each trial and space separately (UCM and ORT). The further analyses (see below) focused on whether PR, PIO, or PII were larger than PB for all trials and both spaces, to assess the overlap in joint angle configurations of the baseline condition and the other conditions.

\section{Statistical analysis}

Variable Error, Movement Time, and Symmetry Index of Velocity were submitted to repeated measures ANOVAs with Condition (R, IS, IO, II) and Distance (20, 30, $40 \mathrm{~cm}$ ) as within-participant factors. This analysis was followed by two planned comparisons whether the deceleration tail was longer in interception compared to reaching, and the angle-of-approach effect in interception. The first preplanned contrast compared reaching to all three interception conditions and the second preplanned contrast compared IO to II.

To assess whether joint angle configurations differed at movement initiation, we conducted a MANOVA with the joint angles at time instant $1 \%$ as dependent variables (shoulder plane of elevation, shoulder elevation, shoulder inward-outward rotation, elbow flexion-extension, forearm pronation-supination, wrist flexion-extension, wrist abduction-adduction, indexfinger flexion-extension, and index-finger abduction-adduction), and Condition (R, IS, IO, II) and Distance $(20,30,40 \mathrm{~cm})$ as within participant factors.

To check whether joint angle variances differed at movement initiation, $\mathrm{V}_{\mathrm{ucm}}(\mathrm{log})$, and $\mathrm{V}_{\text {ort(log) }}$ at time instant $1 \%$ were submitted to separate repeated measures ANOVAs with Condition (R, IS, IO, II) as within participant factor (we averaged across distances). To assess whether joint angles were organized in synergies, $V_{\text {ucm(log), and }} V_{\text {ort(log) }}$ were submitted to a repeated measures ANOVA with Type of Variance $\left(\mathrm{V}_{\mathrm{ucm}(\log )}, \mathrm{V}_{\text {ort }(\log )}\right)$ and Condition $(\mathrm{R}, \mathrm{IS}, \mathrm{IO}$, II) as within participant factors. Note that the experiment also comprised the conditions distance and instant. However, as no effects were expected in the UCM analysis of these conditions, we 


\section{TASK CONSTRAINT ACT AT SYNERGIES AND END-EFFECTOR KINEMATICS}

averaged across distances $(20,30,40 \mathrm{~cm})$ and time instants $(1,10,20,30,40,50,60,70,80$, $90,100 \%$ ) before statistical analysis.

To compare the joint angle configurations reflecting synergies across conditions, we first examined the averages. We calculated the percentage of instances where PIOA, PIIA, or PRA were higher than PB across instances in time $(1,10,20,30,40,50,60,70,80,90,100 \%)$, separately for both spaces (UCM, ORT) and for the three distances. Second, we focused on individual trials. We calculated the percentage of instances where PIO, PII, or PR were larger than PB across all trials and instances $(1,10,20,30,40,50,60,70,80,90,100 \%)$, separately for both spaces (UCM, ORT) and for the three distances. This was done to get a more detailed view on the overlap in joint angle configurations used between different conditions.

Effect sizes were calculated using generalized eta-squared statistics and interpreted as recommended: 0.02 for a small intensity effect, 0.13 for a medium intensity effect, and 0.26 for a large intensity effect (Bakeman, 2005). Significant results with effect sizes smaller than 0.02 are not discussed. Where no planned comparisons were performed and further analyses were appropriate, significant $(p<0.05)$ main effects and interactions were analyzed using NewmanKeuls post-hoc tests.

\section{Results}

Due to (partial) occlusion of the markers or too early initiation of the movement in interception (prior to $150 \mathrm{~ms}$ after the goal target started moving), 3.5\% percent of the trials were discarded. Interception performance was fairly good, with $82.5 \%$ of the targets being intercepted. All other dependent variables, including VE, were computed on intercepted trials only $^{1}$. As a result, the analyses concerned 1268 of the total 1350 trials in the reaching task and 3247 of the total 4050 trials in the interception task.

Even though Variable Error varied to a certain extent between conditions, its magnitude consistently remained small with respect to the task requirements, indicating that overall 


\section{TASK CONSTRAINT ACT AT SYNERGIES AND END-EFFECTOR KINEMATICS}

movements were performed quite accurately. With an overall average of $0.7 \pm 0.04 \mathrm{~cm}(\mathrm{SD})$, Variable Error was small relative to the available $3-\mathrm{cm}$ margin at the target. The ANOVA on Variable Error nevertheless revealed significant (but small intensity) main effects of Condition $\left(F(3,42)=2.83, p=0.049, \eta_{\mathrm{g}}{ }^{2}=0.08\right)$ and Distance $\left(F(2,28)=5.41, p=0.01, \eta_{\mathrm{g}}{ }^{2}=0.03\right)$, as well as a significant interaction of the two $\left(F(6,84)=2.48, p=0.029, \eta_{\mathrm{g}}{ }^{2}=0.05\right)$. The interaction indicated that the differences in Variable Error across the tasks were more pronounced at a target distance of $20 \mathrm{~cm}$ and $40 \mathrm{~cm}$ than at a distance of $30 \mathrm{~cm}$.

\section{Kinematics}

The overall average of Movement Time across all trials was $0.65 \pm 0.01 \mathrm{~s}$ (SD). The ANOVA on Movement Time revealed significant main effects of Condition $(F(3,42)=106.08$, $\left.p<0.001, \eta_{\mathrm{g}}{ }^{2}=0.78\right)$ and Distance $\left(F(2,28)=132.89, p<0.001, \eta_{\mathrm{g}}{ }^{2}=0.11\right)$, and a significant interaction effect of the two $\left(F(6,84)=18.48, p<0.001, \eta_{\mathrm{g}}{ }^{2}=0.05\right)$. Post hoc tests on the interaction revealed that Movement Time was shorter in reaching ( $0.48 \mathrm{~s})$ compared to all interception conditions (IS: $0.73 \mathrm{~s}$; IO: $0.71 \mathrm{~s}$; II: $0.72 \mathrm{~s} ; p$ 's $<0.001$ ). In addition, in reaching Movement Time increased with distance $(20 \mathrm{~cm}: 0.43 \mathrm{~s} ; 30 \mathrm{~cm}: 0.49 \mathrm{~s} ; 40 \mathrm{~cm}: 0.52 \mathrm{~s} ; p$ 's < 0.05) whereas in interception Movement Time was not affected by distance.

The ANOVA on Symmetry Index of Velocity (see Figure 3; overall average 0.43, SD 0.07) revealed a significant main effect of Condition $\left(F(3,42)=34.58, \mathrm{p}<0.001, \eta_{\mathrm{g}}{ }^{2}=0.4\right)$ and Distance $\left(\mathrm{F}(2,28)=20.78, \mathrm{p}<0.001, \eta_{\mathrm{g}}^{2}=0.06\right)$, and an interaction effect of the two $(\mathrm{F}(6,84)$ $\left.=6.24, \mathrm{p}<0.001, \eta_{\mathrm{g}}{ }^{2}=0.02\right)$. The preplanned contrast comparing reaching to interception revealed that peak velocity occurred earlier in interception compared to reaching (R: 0.51 ; I: $0.41 ; p<0.001)$, implying that the velocity patterns in interception had a longer decelerative tail. The preplanned contrast comparing IO and II revealed that peak velocity occurred later in the IO than II condition (IO: 0.42 ; II: $0.40, p=0.016$ ), indicating an angle-of-approach effect. 

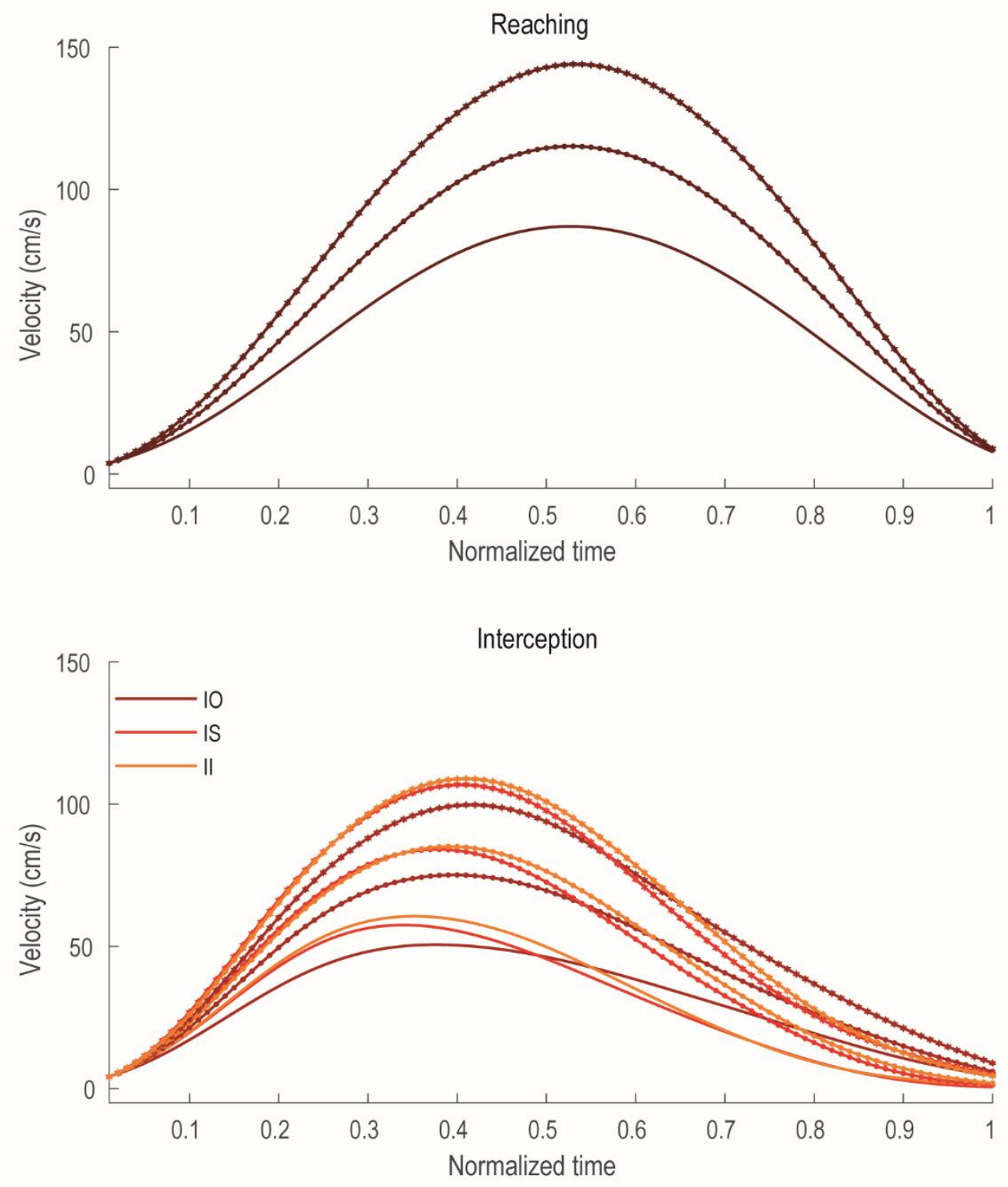

Figure 3. Mean velocity patterns of all conditions. Panel A shows the mean velocity patterns of each reaching condition. The solid lines correspond to the $20 \mathrm{~cm}$ distance, the lightly dotted lines to the $30 \mathrm{~cm}$ distance, and the dotted lines to the $40 \mathrm{~cm}$ distance. Panel B shows the mean velocity patterns of the interception condition. $\mathrm{R}$ is reaching, IS is interception straight, IO is interception outward and II is interception inward.

\section{Synergistic organization of joint angles: UCM analysis}




\section{TASK CONSTRAINT ACT AT SYNERGIES AND END-EFFECTOR KINEMATICS}

Before we turn to the synergistic organization of DOF analysis, we first give a brief indication of the postures used in the experiment and we present how the variables of the UCM analysis evolve over the course of the movement. Figure 4 presents the mean joint angle configurations for reaching and interception of one representative participant. The data for reaching is averaged across distances and data for interception is averaged across distances and IS, IO, and II. Note that the y-axis is scaled differently for each of the subplots of one angle. The figure shows that in the average joint angle trajectories differences between reaching and interception are minimal. Note, the scales correspond to minimum to maximum observed values of averages. The starting posture is with the flexed elbow pointing backwards and the wrist neutral with respect to flexion-extension and with a radial deviation. Over the course of the movement the upper arm is lifted and moves towards the frontal plane. The elbow and the wrist are extended with more radial deviation in the wrist (adduction), to move the arm laterally. The finger gets less adducted over the course of the movement. Additionally, the MANOVA assessing the average joint angles at movement initiation revealed no effects of Distance or Condition for any of the nine joint angles, implying that the starting postures across conditions did not differ.

Figure 5 presents how the variables of the UCM analysis evolve over the course of the movement for each of the conditions. As can be seen in Figure 5, $\mathrm{V}_{\mathrm{ucm}}$ is always considerably higher than $\mathrm{V}_{\text {ort}}$, in all conditions. Over the course of the movement there is some variation in $\mathrm{V}_{\mathrm{ucm}}$ and $\mathrm{V}_{\text {ort }}$, but this variation is relatively small. The ANOVAs on $\mathrm{V}_{\mathrm{ucm}}$ and $\mathrm{V}_{\text {ort }}$ at movement initiation did not reach significance for the main effect of Condition (note that in both analyses the p-value was 0.076), indicating that the joint angle variance does not differ between conditions at movement initiation.

Averaged across time instances, the ANOVA on $V_{\text {ucm }}$ and $V_{\text {ort }}$ (see Figure 6) revealed a significant main effect of Type of Variance $\left(F(1,14)=390.83, p<0.001, \eta_{\mathrm{g}}{ }^{2}=0.91\right)$ and a 


\section{TASK CONSTRAINT ACT AT SYNERGIES AND END-EFFECTOR KINEMATICS}

significant main effect of Condition $\left(F(3,42)=11.52, p<0.001, \eta_{\mathrm{g}}{ }^{2}=0.23\right)$. The main effect of Type of Variance indicated that $\mathrm{V}_{\text {ucm }}$ was always larger than $\mathrm{V}_{\text {ort }}$, which implies DOF were organized in synergies in all experimental conditions. Type of Variance did not interact with Condition implying that the degree of co-variation did not differ amongst conditions. Post hoc tests on the main effect of Condition revealed that both types of variance were higher in all interception conditions compared to reaching ( $p$ 's $<=0.001)$, indicating that the joint angle variance is higher in interception. 

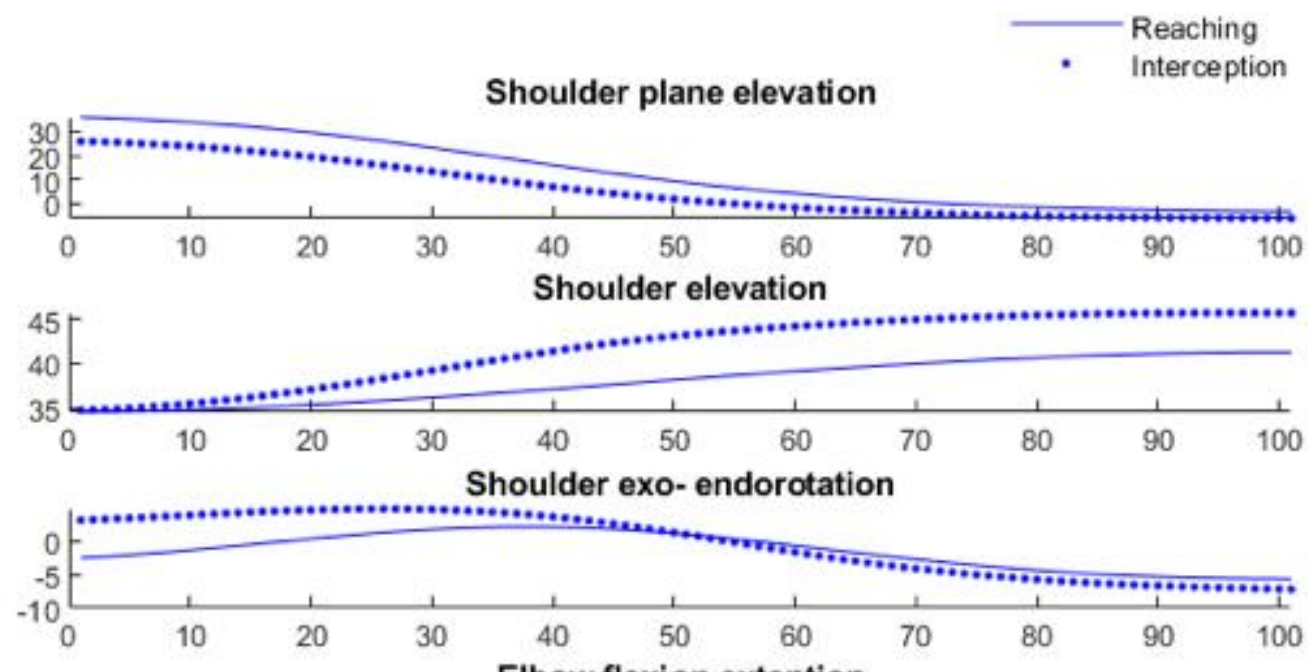

Elbow flexion extention
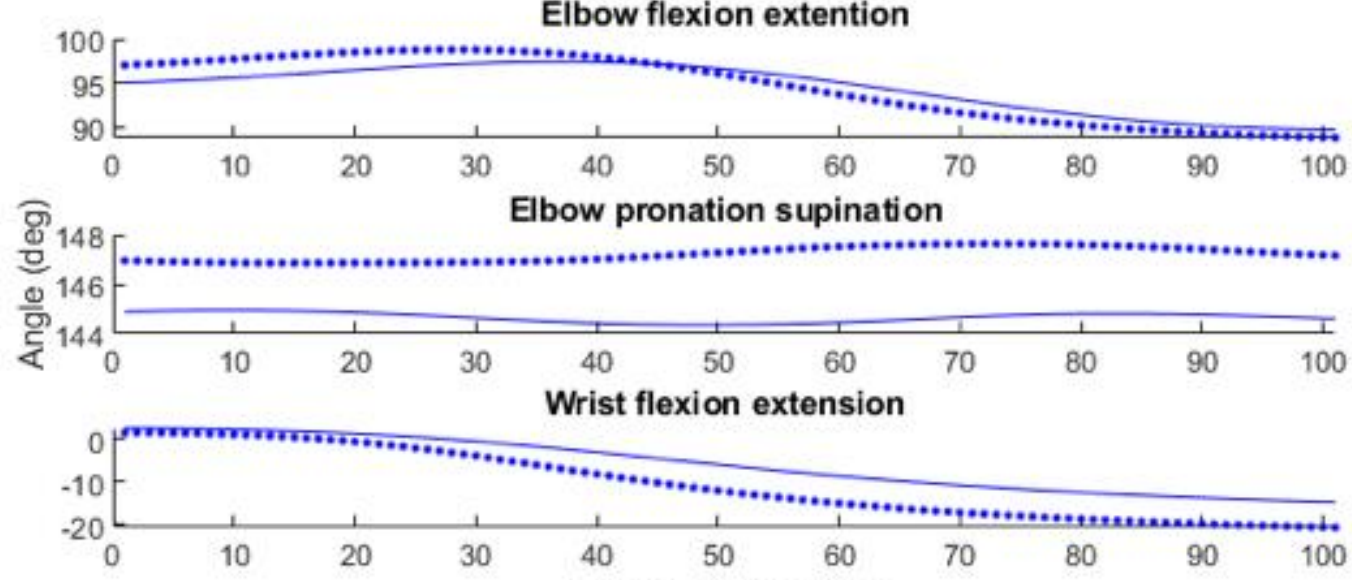

Wrist ab-adduction
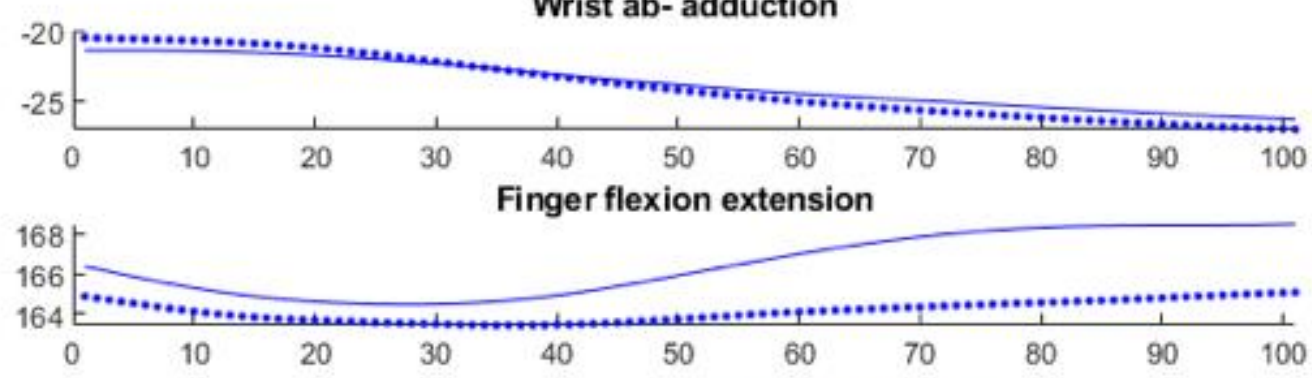

Finger $a b$ - adduction

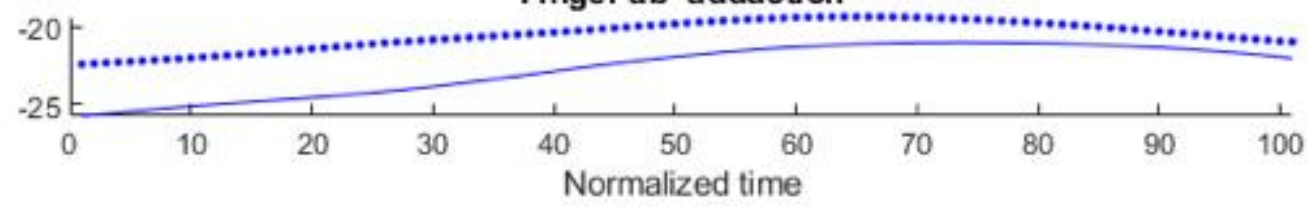

Figure 4. Joint Angle Configuration patterns over time. This is an example of the mean joint angle configurations of a representative participant averaged across trails, distance conditions and for interception also across interception conditions. Note that the y-axis of each subplot of 
one angle is scaled differently, therefore, some joint angles appear to show considerable movement while actually this is only 2-3 degrees.

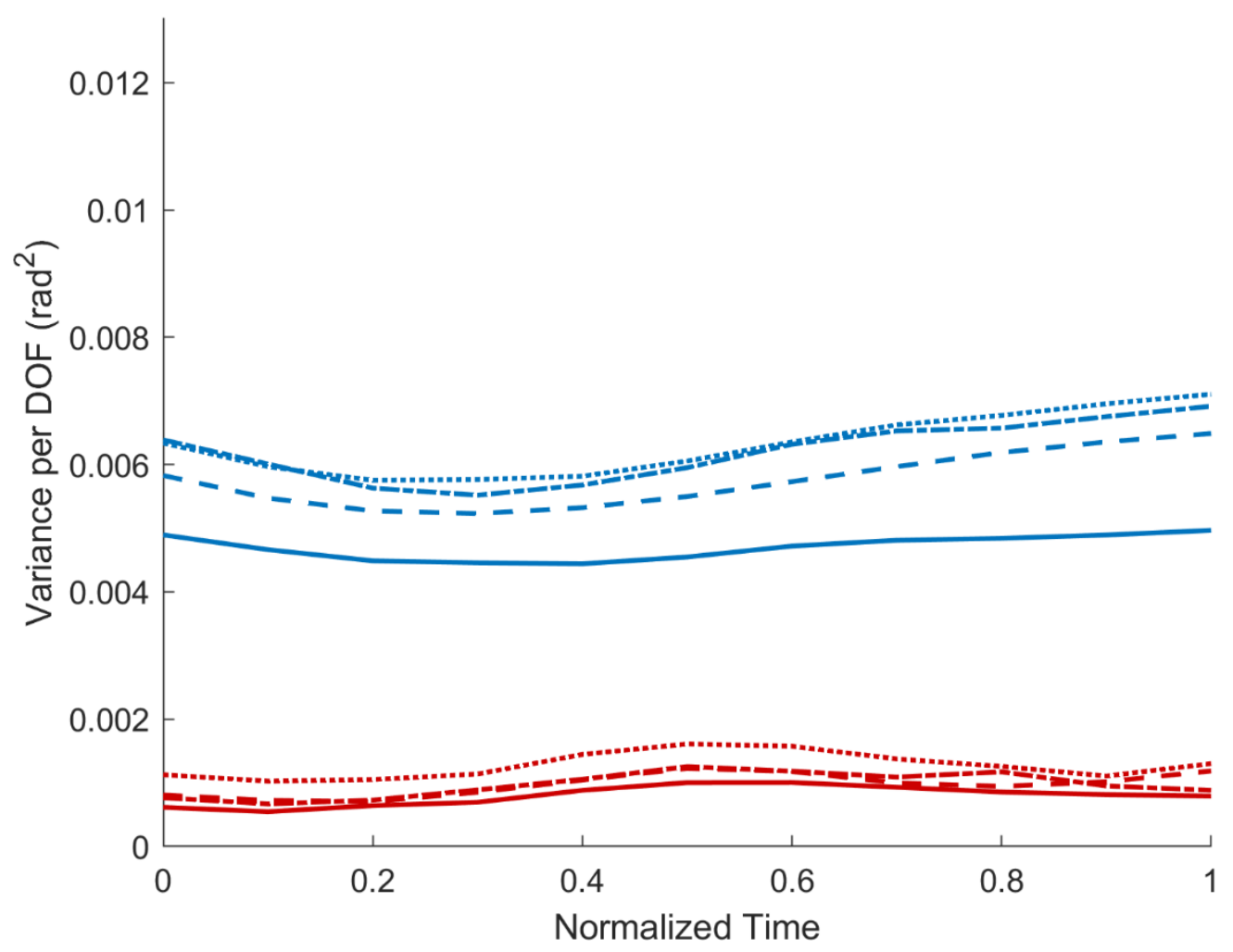

Figure 5. Joint Angle Variance patterns over time. Averages across all participants are depicted in the figure. Solid lines are reaching, dashed lines are interception straight, dashed dotted lines are interception outward and dotted lines are interception inward. The blue (grey) lines are $\mathrm{V}_{\text {ucm }}$ and the red (dark grey) lines are $\mathrm{V}_{\text {ort }}$. 


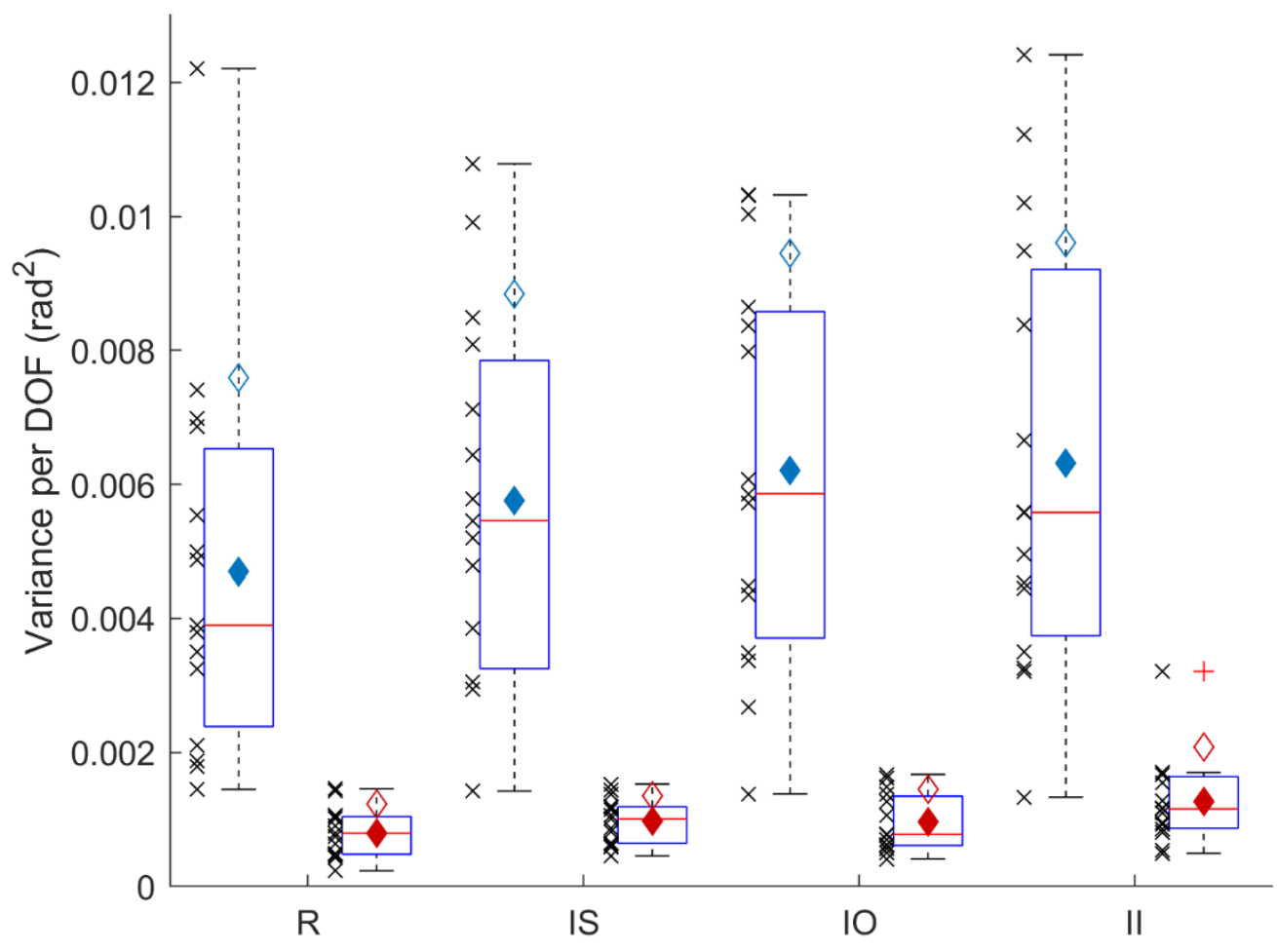

Figure 6. Descriptive statistics of Joint Angle Variance. Boxplots with medians, means, standard deviations and all individual data of participants. The red line represents the median, the diamond shaped filled markers represent the means, the diamond shaped markers represent the standard deviations of the mean, and the black crosses represent the individual data of all participants. Blue (dark grey) represents $\mathrm{V}_{\mathrm{ucm}}$ and red (grey) represents $\mathrm{V}_{\text {ort. }} \mathrm{R}$ is reaching, IS is interception straight, IO is interception outward and II is interception inward. Note that the red plus sign represents an outlier in the data, which is similar to the cross next to it representing this data point.

\section{Clusters of joint angle configurations}

The first analysis for comparing clusters of joint angle configurations was the average JDV analysis comparing the upper bound of the confidence interval of the base condition (PB computed from interception straight condition) to the average JDVs of the other conditions 


\section{TASK CONSTRAINT ACT AT SYNERGIES AND END-EFFECTOR KINEMATICS}

(PIOA, PIIA, PRA). For the interception conditions, PIOA and PIIA were not higher than PB in UCM space, whereas for ORT space PIIA was not higher than PB but PIOA was higher than PB in 1\% of instances (see Table 1 and Figure 7; compare areas to dashed lines). Regarding reaching, PRA was higher than PB in $37.17 \%$ of all instances in UCM space and $53.54 \%$ of all instances averaged in ORT space (see Table 1 and Figure 7; compare areas to solid lines).

The analyses on the clusters of joint angle configurations were done on time-normalized data, in line with the analysis on UCM variables that follows common practices in the literature. However, the analyses on the kinematic patterns showed that the velocity profiles differ amongst conditions and this might confound results based on time-normalized data. Therefore, we checked the clusters of joint angle configurations with spatial-normalized data. Note that spatial-normalized data would not be affected by differences in variables that result from timedependent properties of the variables. The results on spatial-normalized data revealed similar patterns as the results on time-normalized data. To prevent repetition of results, we illustrate our findings in Figure 8, which depicts an example of a representative participant where the averaged differences in projection lengths are shown but now with spatial-normalized data. The comparison of Figure 8 to Figure 7 implies that the dependence of projection lengths on condition does not depend on the type of normalization, time-normalized (Figure 7) or spatialnormalized (Figure 8).

The second analysis for comparing clusters of joint angle configurations was the JDV analysis per trial comparing the upper bound of the confidence interval of the base condition (PB computed from interception straight condition) to JDVs of the other conditions, but now per trial (PIO, PII, PR). The percentage of trials in which the JDV of the individual trials is higher than the upper bound of the confidence interval of the base conditions, gives an indication of the non-overlap region of joint angle configurations between conditions. For the interception conditions, PIO and PII were higher than PB in $36.07 \%$ of all trials and instances 


\section{TASK CONSTRAINT ACT AT SYNERGIES AND END-EFFECTOR KINEMATICS}

in UCM space, whereas for ORT space PII and PIO were higher than PB in $43.23 \%$ of all trials and instances (see Table 2 and Figure 9; compare areas to lines). Regarding reaching, PR was higher than PB in $71.68 \%$ of all trials and instances in UCM space and $80.03 \%$ of all trials and instances averaged in ORT space (Table 2 and Figure 9).

Taking the analyses on the average JDV and the JDV of individual trials together the following picture emerges. Overall, the results does not differ that much for UCM space and ORT space. Based on the averages, one would conclude that interception and baseline conditions do not differ with respect to joint angle configurations used, whereas there is a moderate difference between reaching and interception. The analysis on the individual trials showed a more nuanced picture, where the non-overlap region between reaching and the baseline interception condition is quite large. The non-overlap regions between interception conditions and the baseline interception condition is about one third, which we interpret to be small. Therefore, the joint angle configurations among interception conditions differ not that much whereas the joint angle configurations between reaching and interception are quite different. This suggests overlap in synergies among different interception conditions and substantial non-overlap in synergies between reaching and interception.

Table 1. Descriptive statistics of clusters of joint angle configurations averaged.

\begin{tabular}{|l|l|l|}
\hline Condition & $\begin{array}{l}\text { UCM Space (\%) } \\
\text { Mean (SD) }\end{array}$ & $\begin{array}{l}\text { ORT Space (\%) } \\
\text { Mean (SD) }\end{array}$ \\
\hline IO $20 \mathrm{~cm}$ & $0(0)$ & $0(0)$ \\
\hline IO $30 \mathrm{~cm}$ & $0(0)$ & $1.21(4.69)$ \\
\hline IO $40 \mathrm{~cm}$ & $0(0)$ & $1.82(3.76)$ \\
\hline II $20 \mathrm{~cm}$ & $0(0)$ & $0(0)$ \\
\hline II $30 \mathrm{~cm}$ & $0(0)$ & $0(0)$ \\
\hline II $40 \mathrm{~cm}$ & $0(0)$ & $0(0)$ \\
\hline R $20 \mathrm{~cm}$ & $32.72(40.19)$ & $50.91(33.81)$ \\
\hline
\end{tabular}




\begin{tabular}{|l|l|l|}
\hline R $30 \mathrm{~cm}$ & $35.15(35.85)$ & $55.15(30.43)$ \\
\hline R $40 \mathrm{~cm}$ & $43.64(38.75)$ & $54.55(32.78)$ \\
\hline
\end{tabular}

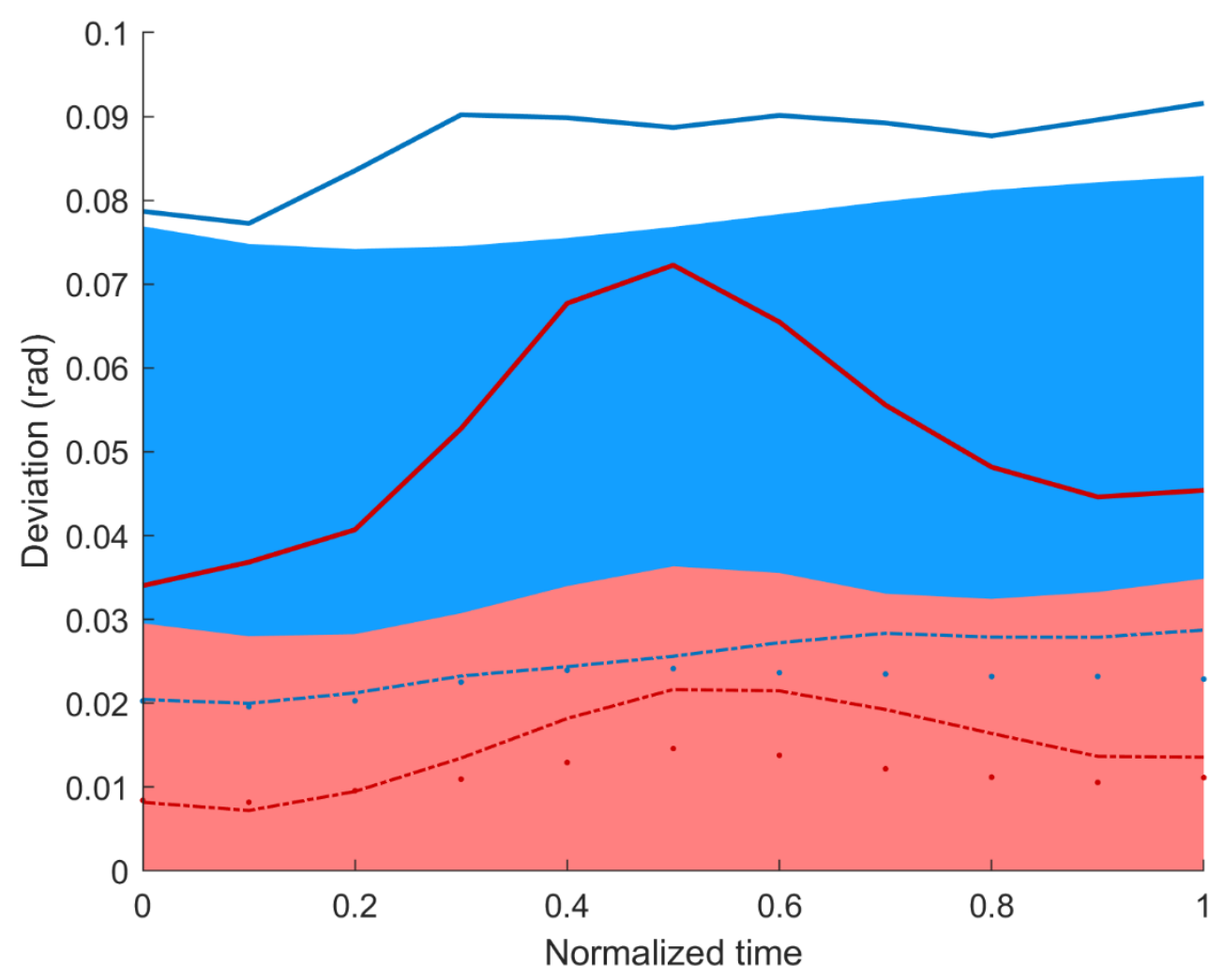

Figure 7. Mean Joint Vector Projection Patterns. The blue (dark grey) lines are Projection ucm $_{\text {. }}$

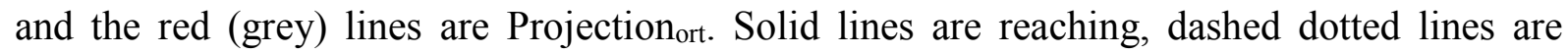
interception outward, and dots are interception inward. The area depicts the area under the upper bound of the CI interval of the base condition interception straight in blue for UCM space and in red for ORT space. 


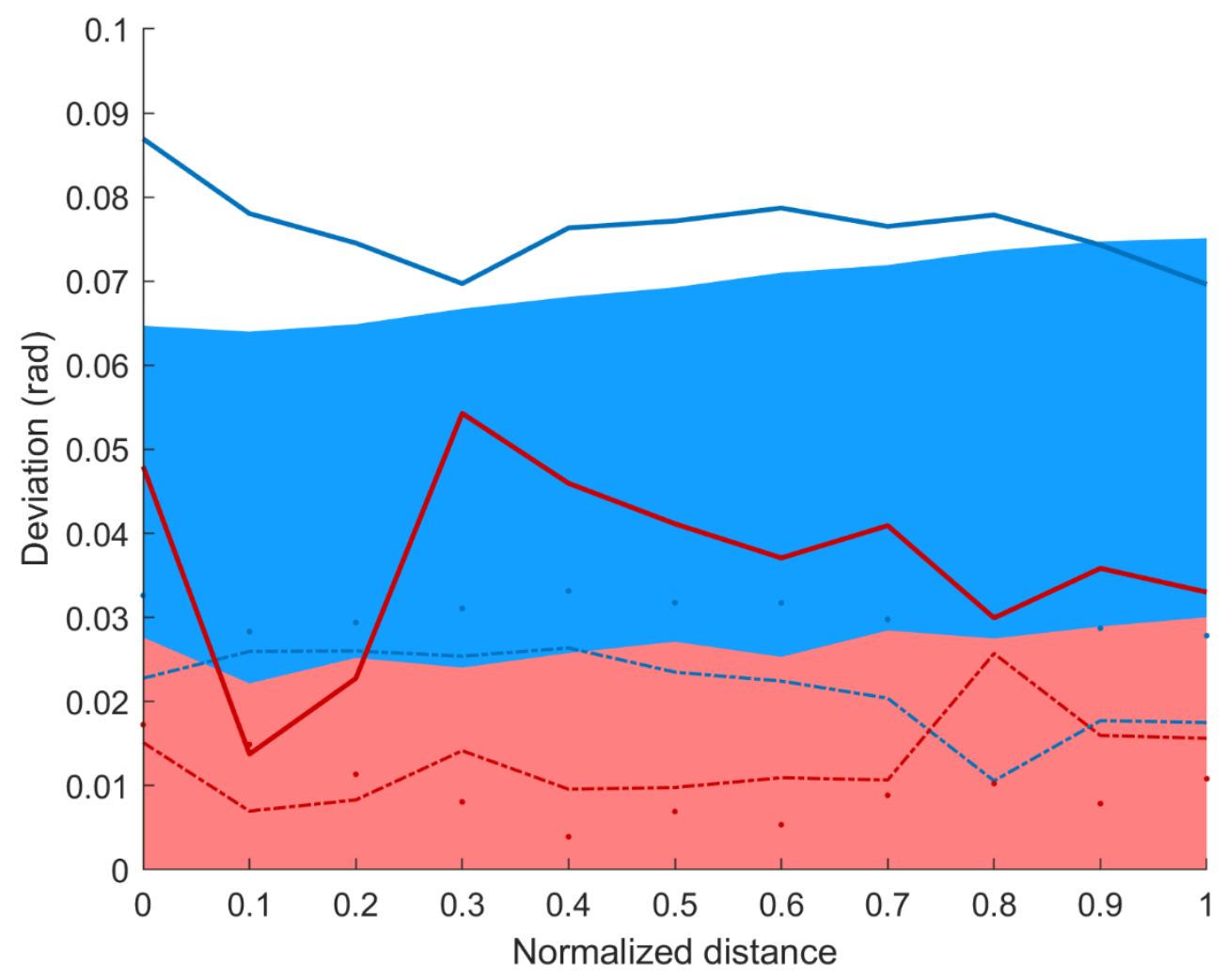

Figure 8. Mean Joint Vector Projection Patterns spatial-normalized. This is an example of a representative participant. The blue (dark grey) lines are Projection ${ }_{\mathrm{ucm}}$ and the red (grey) lines are Projection ${ }_{\text {ort. }}$ The area depicts the area under the upper bound of the CI interval of the base condition interception straight in blue for UCM space and in red for ORT space. Solid lines are reaching, dashed dotted lines are interception outward, and dots are interception inward.

Table 2. Descriptive statistics of clusters of joint angle configurations per trial.

\begin{tabular}{|l|l|l|}
\hline Condition & $\begin{array}{l}\text { UCM Space (\%) } \\
\text { Mean (SD) }\end{array}$ & $\begin{array}{l}\text { ORT Space (\%) } \\
\text { Mean (SD) }\end{array}$ \\
\hline IO $20 \mathrm{~cm}$ & $35.73(2.63)$ & $46.04(2.46)$ \\
\hline IO $30 \mathrm{~cm}$ & $35.15(2.39)$ & $44.31(2.3)$ \\
\hline IO $40 \mathrm{~cm}$ & $39.01(2.39)$ & $43.25(3.1)$ \\
\hline II $20 \mathrm{~cm}$ & $32.97(4.28)$ & $41.41(2.29)$ \\
\hline II $30 \mathrm{~cm}$ & $35.87(2.1)$ & $42.89(2.49)$ \\
\hline II $40 \mathrm{~cm}$ & $37.67(2.7)$ & $41.47(2.23)$ \\
\hline
\end{tabular}




\begin{tabular}{|l|l|l|}
\hline R $20 \mathrm{~cm}$ & $67.94(6)$ & $78.57(4.85)$ \\
\hline R $30 \mathrm{~cm}$ & $70.44(5.33)$ & $82.69(2.79)$ \\
\hline R $40 \mathrm{~cm}$ & $76.66(3.84)$ & $78.83(3.77)$ \\
\hline
\end{tabular}
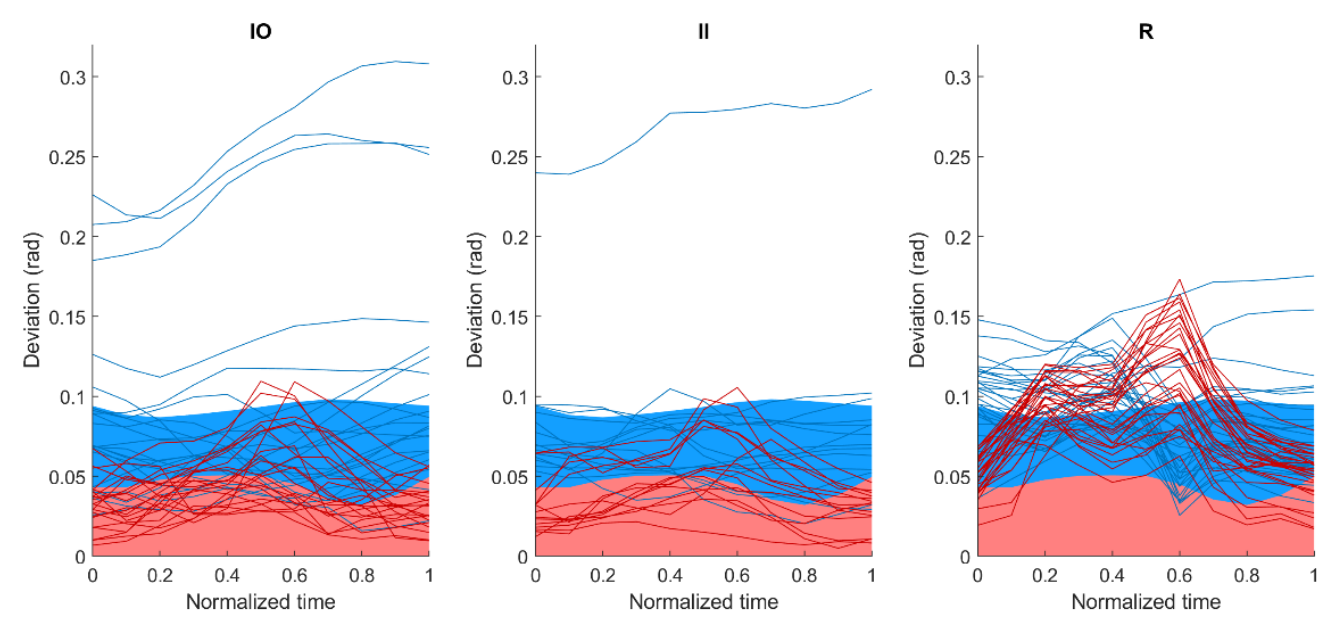

Figure 9. Joint Vector Projection Patterns per trial. This is an example of a representative participant in a certain distance condition. The blue (grey) lines are Projection $\mathrm{ucm}_{\mathrm{u}}$ and the red (dark grey) lines are Projection ${ }_{\text {ort }}$ Each line represents a trial of the condition. The area depicts the area under the upper bound of the CI interval of the base condition interception straight in blue for UCM space and in red for ORT space. IO is interception outward, II is interception inward and $\mathrm{R}$ is reaching.

\section{Discussion}

The current study focused on emergent synergies and aimed to establish whether different synergies emerge as a function of changes in task constraints. Synergies were characterized by the degree of co-variation amongst joint angles across repetitions and by the cluster of joint angle configurations employed by the synergy. This characterization of synergies reflects the organization of joint-angles in a unit to stabilize task performance while at the same time appreciating the specific joint angle configurations used to perform a task. Synergies can change if task constraints vary, and we assumed that task constraints affect synergies and end-effector 


\section{TASK CONSTRAINT ACT AT SYNERGIES AND END-EFFECTOR KINEMATICS}

kinematics independently, as proposed by Kay (1988). To determine which variations in task constraints affected synergies only, we examined both synergies and end-effector kinematics. To do this, we used different conditions of manual reaching and manual interception in our experiment of which differences in end-effector kinematics could be expected based on earlier studies (e.g., compare Morasso, 1981 to Ledouit et al., 2013). Our findings confirmed these differences, demonstrating that the velocity profile of the end-effector was roughly symmetric in manual reaching, whereas in manual lateral interception it had a longer decelerative tail. In addition, the velocity profile showed an angle-of-approach effect in interception. Subsequently, our results demonstrated that the joint angle configurations exhibited primarily co-variation $\left(\mathrm{V}_{\mathrm{ucm}}\right)$ instead of other variation $\left(\mathrm{V}_{\text {ort }}\right)$ in all conditions, indicating that joint angles were organized in synergies. Note that we established that the starting posture and the variability in joint angles at start of the movement was not different between conditions. The key finding of the current paper is that amongst interception conditions the clusters of joint angle configurations showed large overlap, whereas between reaching and interception the employed clusters of joint angle configurations only partly overlapped. In the current study this was interpreted as that emergent synergies of reaching and interception employed different postural configurations in the arm, whereas amongst interception conditions similar synergies emerged. Taken together, these results imply that some task constraints (related to for example whether the target moves or not, and whether the end-effector has to stop in the target) operate at the level of synergies while other task constraints (for instance different trajectories of to-beintercepted objects) only affect kinematics, which is in line with the suggestion of Kay (1988). That is, the interaction of constraint leads to the production of goal-directed behavior, but, depending on the specifics of the constraints in some cases different synergies emerge while in

other cases a similar synergy is constrained differently, both producing various kinematic patterns at the end-effector level. 


\section{TASK CONSTRAINT ACT AT SYNERGIES AND END-EFFECTOR KINEMATICS}

Our findings regarding the end-effector kinematics are in line with the literature. As in previous studies (Flash \& Hogan, 1985; Gordon et al., 1994; Morasso, 1981) we found that the velocity profile in reaching is roughly bell-shaped. Furthermore, the velocity profile in manual interception was asymmetric with a longer deceleration tail and showed an angle-of-approach effect, an effect also established in earlier studies (Ledouit et al., 2013; Michaels et al., 2006; Montagne et al., 1999; Peper et al., 1994), implying systematic differences between targets moving inward (i.e., with the lateral component of the target trajectory moving toward the participant) and moving outward (i.e., with the lateral component of the target trajectory moving away from the participant). More specifically, the moment of peak velocity occurred earlier for the inward moving targets compared to the outward moving targets. All our findings regarding the kinematics of the end-effector are concurrent with earlier findings (Ledouit et al., 2013; Michaels et al., 2006; Montagne et al., 1999; Peper et al., 1994), however, the current paper is to our knowledge the first to make a direct end-effector kinematics comparison between reaching and interception, and confirms the presumed differences.

With respect to the synergistic organization of joint angles, ample literature has revealed that in manual reaching $\mathrm{V}_{\text {ucm }}$ was larger than $\mathrm{V}_{\text {ort }}$ (see also Black, Riley, \& McCord, 2007; Domkin, Laczko, Djupsjöbacka, Jaric, \& Latash, 2005; Golenia et al., 2018; Greve et al., 2013; Hansen et al., 2015; Krüger et al., 2013; Romero, Kallen, Riley, \& Richardson, 2015; Scholz \& Schöner, 1999; Tseng et al., 2003; Valk et al., 2016; Van Der Steen \& Bongers, 2011). This was also revealed in our reaching conditions, implying that DOF were organized in synergies. A novel contribution to the literature is that $\mathrm{V}_{\text {ucm }}$ was also larger than $\mathrm{V}_{\text {ort }}$ in a manual lateral interception task. This supports earlier findings indicating that joint angles are coordinated in synergies in unrestrained catching (Bockemühl et al., 2010; Mazyn et al., 2006), using other methods of analysis than a UCM analysis. Moreover, we found that both $\mathrm{V}_{\mathrm{ucm}}$ and $\mathrm{V}_{\text {ort }}$ were higher in interception compared to reaching, showing that joint angle coordination patterns 


\section{TASK CONSTRAINT ACT AT SYNERGIES AND END-EFFECTOR KINEMATICS}

were more variable in interception than in reaching. Importantly, the type of variance (i.e., $\mathrm{V}_{\mathrm{ucm}}$ and $\mathrm{V}_{\text {ort }}$ ) did not interact with other factors, which indicated that the degree of co-variation did not differ amongst conditions and tasks, implying that the coupling strength among DOF within the synergies of those tasks did not differ.

Our most important finding was that some task constraints (i.e., differences between reaching and interception) affected synergies, whereas other task constraints (i.e., angle of approach) did not affect synergies but were involved in constraining synergies, thereby affecting end-effector kinematics. This finding is based on the non-overlap regions in clusters of joint angle configurations between reaching and interception, whereas clusters of joint angle configurations fully overlapped amongst interception conditions. Interestingly, the end-effector kinematics did differ between reaching and interception and amongst interception conditions with different angles of approach. We interpret these findings as support for the two-step process of constraining DOF as proposed by Kay (1988). Kay argued that the emergence of a synergy is the first step in the constraining process. This synergy takes the form of a dynamical system (Kelso, 1995; Kugler \& Turvey, 1987.; Kugler, Kelso, \& Turvey, 1980; Saltzman \& Kelso, 1987), of which the properties are characterized by the specifics of an attractor. An attractor can be defined as a set of states where a system tends to evolve towards. Such an attractor generates a cluster of joint angle configurations with a certain size and location in joint space that reflect the synergy, as is shown in Figure 1B. The second step of the process is the additional constraining of the attractor leading to the production of the actual movement (Kay, 1988). It has also been suggested that this two-step process is at play in interpersonal coordination (Riley et al., 2011; Romero et al., 2015), suggesting that this process coordinates DOF at different behavioral levels.

Some might argue that the differences in synergies between reaching and interception stem from the differences in movement times and differences in the velocity profile of the end- 


\section{TASK CONSTRAINT ACT AT SYNERGIES AND END-EFFECTOR KINEMATICS}

effector. That is, if the movement times and velocity profiles differ between reaching and interception then the postural configurations would have to differ. However, we argue that the different synergy is a finding in itself. Using a spatial normalization of the end-effector trajectory and the joint angles, where time is 'removed' from the analyses, our findings were similar to our findings with time normalization. The similarity in results with different normalizations strengthens the validity of our conclusions. Alternative ways to test our conclusions would be to change or add task constraints to make the movement times of reaching and interception more alike, and examine whether different synergies still show up in reaching and interception. However, strategies to do this, such as imposing movement times or changing target sizes would substantially affect the kinematic profiles (cf. Bongers, Fernandez, \& Bootsma, 2009; Mottet \& Bootsma, 1999). This would imply that the adding or manipulating constraints would introduce a specific shape of the velocity profile creating other differences between conditions, while the idea was that these manipulations of constraints would remove differences in kinematics. In other words, these changes of constraints would throw out the baby with the bathwater. Therefore, we think that the similar findings for time and spatial normalizations support our conclusions and that attempts to make movement times and velocity profiles more the same have fundamental problems.

To evaluate the validity of our interpretation of the results we now turn to two alternative interpretations of synergies, and relate them to our results. One approach that is relevant in this respect is the approach of Latash and colleagues (Gorniak, Zatsiorsky, \& Latash, 2007a, 2007b, 2009a, 2009b) who employ the UCM approach to assess hierarchical control of synergies. Imagine grasping an object and holding it between the thumb and the four fingers. In this scenario the thumb and the fingers produce forces with opposite direction on the hand-held object. To stabilize the object in the hand, the force of the thumb needs to have the same magnitude but has to be in opposite direction as the combined force of the fingers. Hence, there 


\section{TASK CONSTRAINT ACT AT SYNERGIES AND END-EFFECTOR KINEMATICS}

needs to be a synergy between the thumb and the fingers as a group. But the four fingers can produce the total combined force of the fingers in different combinations, that is, the forces of the individual fingers co-vary to produce the combined finger force. Therefore, the four fingers represent a second synergy. These synergies (i.e., thumb-combined fingers and four fingers) are hierarchically related, and depending on conditions (i.e., using multiple fingers of two hands, or using a bimanual grasp with two persons; Gorniak et al. 2007a; 2009b) more or less co-variation between the DOF in the two synergies were found. More specifically, it was shown that if the synergy that is higher in the hierarchy (i.e., the thumb-combined fingers synergy) becomes more important, the lower synergy disappears (Gorniak et al., 2009b). The difference between the two synergies becomes apparent and the higher synergy becomes more important when task constraints are more challenging. The hierarchical synergies show that task constraints affect synergies of which the set of DOF (i.e., thumb-combined fingers versus four fingers) in the synergies differ. In our experiment we show that synergies also change if task constraints change on the same set of DOF (i.e., the joint angles in the arm). Hence, combining these different perspectives on synergies seems to suggest that linkages between DOF to form synergies are nested in different layers of organization that are all affected by different types of constraints.

The second influential approach on the organization of DOF in synergies is the muscle synergies perspective (cf. Bizzi, Cheung, d'Avella, Saltiel, \& Tresch, 2008; Bizzi \& Cheung, 2013; d'Avella, Saltiel, \& Bizzi, 2003; Tresch \& Jarc, 2010). Muscle synergies are modules in the neuromotor system where each module activates a certain set of muscles in a fixed proportional way. To produce a movement the neuromotor system activates these modules over time and varies the activation level of the modules to generate different movements (see Bruton \& O’Dwyer, 2018 for an recent in-depth overview). Several studies within this approach have focused on reaching (e.g. D’Avella, Fernandez, Portone, \& Lacquaniti, 2008; D’Avella, 


\section{TASK CONSTRAINT ACT AT SYNERGIES AND END-EFFECTOR KINEMATICS}

Portone, Fernandez, \& Lacquaniti, 2006; d'Avella \& Lacquaniti, 2013; Russo, D’Andola, Portone, Lacquaniti, \& d'Avella, 2014) and revealed that about 4 to 5 muscle synergies can explain the variance in EMG patterns across different conditions. D'Andola et al. (2013) examined one-handed unrestrained ball catching and found that 2 synergies explained the variance in EMG in the arm muscles. The difference in the number of synergies required to explain reaching and one-handed catching most likely originates from the different number of experimental conditions in the different studies. D'Andola et al. (2013) showed that in catching one of the synergies brought the hand to the region where the ball was caught and the other synergy brought the hand to the actual catching location. Although this muscle synergy perspective assumes these muscle synergies to be fixed, the finding of fixed modules is not supported by all studies searching for the building blocks of EMG patterns (e.g. Muceli, Falla, \& Farina, 2014, Tropea, Monaco, Coscia, Posteraro, \& Micera, 2013; Valk, Mouton, Otten, \& Bongers, 2019). That is, several studies searching for the building blocks of EMG patterns show that invariances underlying EMG patterns depend on task constraints pointing to the flexible organization underlying muscle patterns. This flexibility is in line with an organization of DOF in dynamic synergies following from self-organization, as proposed in the current paper. In line with this, it might be interesting for future studies to examine whether structure in variability and clusters of organization can also be found at the muscular level.

Our results illustrate a hallmark of the dynamical systems approach, that is, there is structure in variability that characterizes the attractor reflecting the organization of the DOF (cf. Riley \& Turvey, 2002). In doing so, we studied structure in variability at the level of the joint angles and how it relates to variability at the end-effector level (Latash et al., 2007; cf. Ranganathan \& Newell, 2010; cf. Sternad, 2018). Our analyses focusing on two levels add to the usual dynamical systems analyses that characterizes the attractor at just the end-effector level (Beek, Peper, Stegeman, 1995; Beek, Rikkert, \& van Wieringen, 1996; Haken, Kelso, \& 


\section{TASK CONSTRAINT ACT AT SYNERGIES AND END-EFFECTOR KINEMATICS}

Bunz, 1985; Mottet \& Bootsma, 1999; Schmidt, Carello, \& Turvey, 1990). Coordination at the joint angle level in the arm was also studied from a dynamic systems perspective. That is, Buchanan et al. (1997) analyzed changes in relative phase relations between wrist, elbow, and shoulder joints under systematic variation of curvature of end-effector movements. However, relative phase is computed between two angles. To understand coordination amongst more than two angles as well as the relation between joint angle variability and end-effector variability, a different technique is required. For that reason, we followed studies in interpersonal coordination (Riley et al., 2011; Romero et al., 2015) and in motor development (Golenia et al., 2018), and employed the UCM analysis in the current study.

Applying the UCM analysis and its extended techniques to assess coordination amongst more DOF might also be employed to understand how emergent synergies deal with perturbations in future studies. That is, a key example of synergistic organization of DOF is an experiment on speech production where participants were asked to produce a specific syllable, while during the production of this syllable the lower lip was mechanically perturbed (Kelso et al., 1984). Interestingly, during the perturbation the production of the syllable was preserved through instantaneous changes in the movements of the upper lip, lower lip, or tongue, depending on the syllable (i.e., motor equivalence). Over the years, other studies also showed adjustments in DOF other than the one perturbed to maintain the function of the synergy (Abbs

\& Gracco, 1984; Cole \& Abbs, 1987; Kelso et al., 1984; Schettino et al., 2017; Van De Kamp \& Zaal, 2007; Zaal \& Bongers, 2014). Note that these studies focus on a limited number of DOF to understand how the synergy maintains its function after a perturbation. A study employing UCM analyses and one of its extensions, that is, motor equivalence analysis, showed that the collective DOF adjust (i.e., most changes in joint angle configurations were motor equivalent) after a perturbation of the elbow when an elastic band gently pulled at the elbow during reaching (Mattos et al., 2011). This implies that current techniques can examine how the 


\section{TASK CONSTRAINT ACT AT SYNERGIES AND END-EFFECTOR KINEMATICS}

collective DOF are coordinated to maintain the function of the synergy. Exploiting these techniques in future studies would elevate our understanding of the process of the emergence of synergies by understanding how a large set of DOF contributes to meet task demands under perturbations.

With respect to the employed analyses techniques used to examining the cluster of joint angle configurations, we applied a modified version of a UCM based method to analyze motor equivalence (Mattos et al., 2011; Schöner, 2008). Schöner's method to show motor equivalence computed projection lengths of a perturbed condition on the UCM of an unperturbed condition. Actions were considered motor equivalent if those projection lengths were larger in the UCM space compared to the orthogonal space (note that all conditions of the current study were motor equivalent). This analysis, comparing differences in projection length between spaces, inspired our analysis to assess differences in synergies, where we looked at differences in projection lengths within each space separately (i.e., UCM and ORT space, respectively). With this adapted version of the UCM analysis we quantified the location and the size in joint space of a cluster of joint angle configurations, which we take to reflect the joint angle configuration employed by the synergy.

In conclusion, the current study examined whether different synergies emerged when task constraints were varied in manual reaching and a manual interception. We found that although kinematic profiles differed among all conditions, synergies only varied between reaching and interception, but not among interception conditions. Together these results support the two-step process as proposed by Kay (1988) to coordinate the redundant DOF in the action system. 
TASK CONSTRAINT ACT AT SYNERGIES AND END-EFFECTOR KINEMATICS

\section{References}

Abbs, J. H., \& Gracco, V. L. . (1984). Control of Complex Motor Gestures : Orofacial Muscle Responses to Load Perturbations of Lip During Speech. Journal of Neurophysiology, 5(4), $705-723$.

Bakeman, R. (2005). Recommended effect size statistics for repeated measures designs. Behavior Research Methods, 37(3), 379-84. Retrieved from http://www.ncbi.nlm.nih.gov/pubmed/16405133

Beek, Peter J.; Peper, C.E.; Stegeman, D. F. (1995). Dynamical models of movement coordination. Human Movement Science, 14, 573-608.

Beek, P. J., Rikkert, W. E. I., \& van Wieringen, P. C. W. (1996). Limit cycle properties of rhythmic forearm movements. Journal of Experimental Psychology: Human Perception and Performance, 22(5), 1077-1093. Retrieved from papers2://publication/uuid/45A162DE-8228-474F-897E-0996D099BC23

Bernstein, N. (1967). The Coordination and regulation of Movements. Oxford New York: Pergamon Press; Oxford.

Bizzi, E., \& Cheung, V. C. K. (2013). The neural origin of muscle synergies. Frontiers in Computational Neuroscience, 7(April), 51. http://doi.org/10.3389/fncom.2013.00051

Bizzi, E., Cheung, V. C. K., d'Avella, A., Saltiel, P., \& Tresch, M. (2008). Combining modules for movement. Brain Research Reviews, 57(1), 125-133. http://doi.org/10.1016/j.brainresrev.2007.08.004

Black, D. P., Riley, M. A., \& McCord, C. K. (2007). Synergies in intra- and interpersonal interlimb rhythmic coordination. Motor Control, 11(4), 348-73. Retrieved from http://www.ncbi.nlm.nih.gov/pubmed/18042965

Bockemühl, T., Troje, N. F., \& Dürr, V. (2010). Inter-joint coupling and joint angle synergies of human catching movements. Human Movement Science, 29(1), 73-93. 


\section{TASK CONSTRAINT ACT AT SYNERGIES AND END-EFFECTOR KINEMATICS}

http://doi.org/10.1016/j.humov.2009.03.003

Bongers, R. M., Fernandez, L., \& Bootsma, R. J. (2009). Linear and logarithmic speed-accuracy trade-offs in reciprocal aiming result from task-specific parameterization of an invariant underlying dynamics. Journal of Experimental Psychology. Human Perception and Performance, 35(5), 1443-1457. http://doi.org/10.1037/a0015783

Bruton, M., \& O’Dwyer, N. (2018). Synergies in coordination: a comprehensive overview of neural, computational and behavioral approaches. Journal of Neurophysiology, jn.00052.2018. http://doi.org/10.1152/jn.00052.2018

Buchanan, J. J., Kelso, J. A. S., \& De Guzman, G. C. (1997). Self-organization of trajectory formation I. Experimental evidence. Biological Cybernetics, 76(4), 257-273. http://doi.org/10.1007/s004220050338

Cole, K. J., \& Abbs, J. H. (1987). Kinematic and electromyographic responses to perturbation of a rapid grasp. Journal of Neurophysiology, 57(5), 1498-1510. http://doi.org/10.1152/jn.1987.57.5.1498

D’Andola, M., Cesqui, B., Portone, A., Fernandez, L., Lacquaniti, F., \& d'Avella, A. (2013). Spatiotemporal characteristics of muscle patterns for ball catching. Frontiers in Computational Neuroscience, 7(August), 1-11. http://doi.org/10.3389/fncom.2013.00107

D’Avella, A., Fernandez, L., Portone, A., \& Lacquaniti, F. (2008). Modulation of phasic and tonic muscle synergies with reaching direction and speed. Journal of Neurophysiology, 100(3), 1433-1454. http://doi.org/10.1152/jn.01377.2007

d'Avella, A., \& Lacquaniti, F. (2013). Control of reaching movements by muscle synergy combinations. Frontiers in Computational Neuroscience, 7(APR 2013), 1-7. http://doi.org/10.3389/fncom.2013.00042

D’Avella, A., Portone, A., Fernandez, L., \& Lacquaniti, F. (2006). Control of fast-reaching movements by muscle synergy combinations. Journal of Neuroscience, 26(30), 7791- 


\section{TASK CONSTRAINT ACT AT SYNERGIES AND END-EFFECTOR KINEMATICS}

7810. http://doi.org/10.1523/JNEUROSCI.0830-06.2006

d'Avella, A., Saltiel, P., \& Bizzi, E. (2003). Combinations of muscle synergies in the construction of a natural motor behavior. Nature Neuroscience, 6(3), 300-308. http://doi.org/10.1038/nn1010

de Freitas, S. M. S. F., Scholz, J. P., \& Stehman, A. J. (2007). Effect of motor planning on use of motor abundance. Neuroscience Letters, 417(December 2006), 66-71. http://doi.org/10.1016/j.neulet.2007.02.037

Domkin, D., Laczko, J., Djupsjöbacka, M., Jaric, S., \& Latash, M. L. (2005). Joint angle variability in 3D bimanual pointing: Uncontrolled manifold analysis. Experimental Brain Research, 163, 44-57. http://doi.org/10.1007/s00221-004-2137-1

Domkin, D., Laczko, J., Jaric, S., Johansson, H., \& Latash, M. L. (2002). Structure of joint variability in bimanual pointing tasks. Experimental Brain Research, 143, 11-23. http://doi.org/10.1007/s00221-001-0944-1

Flash, T., \& Hogan, N. (1985). The coordination of arm movements: an experimentally confirmed mathematical model. The Journal of Neuroscience, 5(7), 1688-1703. http://doi.org/4020415

Golenia, L., Schoemaker, M. M., Otten, E., Mouton, L. J., \& Bongers, R. M. (2018). Development of reaching during mid-childhood from a Developmental Systems perspective. PLoS ONE, 13(2), 1-17. http://doi.org/10.1371/journal.pone.0193463

Golenia, L., Schoemaker, M. M., Otten, E., Tuitert, I., \& Bongers, R. M. (2018). The development of consistency and flexibility in manual pointing during middle childhood. Developmental Psychobiology, 60(5). http://doi.org/10.1002/dev.21741

Gordon, J., Ghilardi, M. F., \& Ghez, C. (1994). Accuracy of planar reaching movements - I. Independence of direction and extent variability. Experimental Brain Research, 99(1), 97111. http://doi.org/10.1007/BF00241415 


\section{TASK CONSTRAINT ACT AT SYNERGIES AND END-EFFECTOR KINEMATICS}

Gorniak, S. L., Zatsiorsky, V. M., \& Latash, M. L. (2007a). Emerging and disappearing synergies in a hierarchically controlled system. Experimental Brain Research, 183(2), 259-270. http://doi.org/10.1007/s00221-007-1042-9

Gorniak, S. L., Zatsiorsky, V. M., \& Latash, M. L. (2007b). Hierarchies of synergies: An example of two-hand, multi-finger tasks. Experimental Brain Research, 179(2), 167-180. http://doi.org/10.1007/s00221-006-0777-z

Gorniak, S. L., Zatsiorsky, V. M., \& Latash, M. L. (2009a). Hierarchical control of static prehension: I. Biomechanics. Experimental Brain Research, 193(4), 615-631. http://doi.org/10.1007/s00221-008-1662-8

Gorniak, S. L., Zatsiorsky, V. M., \& Latash, M. L. (2009b). Hierarchical control of static prehension: II. Multi-digit synergies. Experimental Brain Research, 194(1), 1-15. http://doi.org/10.1007/s00221-008-1663-7

Greene, P. H. (1972). Problems of organization of motor systems. Progress in Theoretical Biology. Academic press, inc. http://doi.org/10.1016/B978-0-12-543102-6.50013-3

Greve, C., Zijlstra, W., Hortobágyi, T., \& Bongers, R. M. (2013). Not All Is Lost: Old Adults Retain Flexibility in Motor Behaviour during Sit-to-Stand. PLoS ONE, 8(10). http://doi.org/10.1371/journal.pone.0077760

Haken, H., Kelso, J. A. S., \& Bunz, H. (1985). A theoretical model of phase transitions in human hand movements. Biological Cybernetics, 51(5), 347-356. http://doi.org/10.1007/BF00336922

Hansen, E., Grimme, B., Reimann, H., \& Schöner, G. (2015). Carry-over coarticulation in joint angles. Experimental Brain Research, 233(9), 2555-2569. http://doi.org/10.1007/s00221$015-4327-4$

Kay, B. A. (1988). The dimensionality of movement trajectories and the degrees of freedom problem: A tutorial. Human Movement Science, 7(87), 343-364. 


\section{TASK CONSTRAINT ACT AT SYNERGIES AND END-EFFECTOR KINEMATICS}

Kelso, J. A. S. (1995). Dynamic patterns: The self-organization of brain and behavior. Cambridge, MA, US: The MIT Press.

Kelso, J. A. S. (2009). Progress in motor control: a multidisciplinary perspective. Springer. http://doi.org/10.1007/978-0-387-77064-2_24

Kelso, J. A. S., Tuller, B., Vatikiotis-Bateson, E., \& Fowler, C. A. (1984). Functionally Specific Articulatory Cooperation Following Jaw Perturbations During Speech: Evidence for Coordinative Structures. Journal of Experimental Psycholgy, Human Perception and Performance, 10(6), 812-832.

Krüger, M., Eggert, T., \& Straube, a. (2013). Age-related differences in the stabilization of important task variables in reaching movements. Motor Control, 17, 313-9. http://doi.org/10.1123/mc.2014-0005

Kugler, P., Turvey, M. (1987). Information, Natural Law, and the Self-Assembly of Rhythmic Movement. Hillsdale, NJ: Erlbaum.

Kugler, P. N. ., Kelso, J. A. S., \& Turvey, M. T. . (1980). On the concept of coordinative structures as dissipative structures: I. Theoretical lines of convergence. In George Stelmach J. Requin (Ed.), Tutorials in Motor Behavio. North-Holland Publishing Company. http://doi.org/10.1016/S0166-4115(08)61936-6

Latash, M. L., Scholz, J. P., \& Schöner, G. (2007). Toward a new theory of motor synergies. Motor Control, 11, 276-308. http://doi.org/17715460

Ledouit, S., Casanova, R., Zaal, F. T. J. M., \& Bootsma, R. J. (2013). Prospective control in catching: The persistent angle-of-approach effect in lateral interception. PLoS ONE, 8(11), 1-11. http://doi.org/10.1371/journal.pone.0080827

Matthew Tresch, \& Anthony Jarc. (2010). The case for and against muscle synergies. Current Opinion in Neurobiology, 19(6), 1-11. http://doi.org/10.1016/j.conb.2009.09.002.The

Mattos, D. J. S., Latash, M. L., Park, E., Kuhl, J., \& Scholz, J. P. (2011). Unpredictable elbow 


\section{TASK CONSTRAINT ACT AT SYNERGIES AND END-EFFECTOR KINEMATICS}

joint perturbation during reaching results in multijoint motor equivalence. Journal of Neurophysiology, 106(3), 1424-1436. http://doi.org/10.1152/jn.00163.2011

Mazyn, L. I. N., Montagne, G., Savelsbergh, G. J. P., \& Lenoir, M. (2006). Reorganization of catching coordination under varying temporal constraints. Motor Control, 10, 143-159.

Michaels, C. F., Jacobs, D. M., \& Bongers, R. M. (2006). Lateral interception II: predicting hand movements. Journal of Experimental Psychology. Human Perception and Performance, 32(2), 459-472. http://doi.org/10.1037/0096-1523.32.2.459

Montagne, G., Laurent, M., Durey, A., \& Bootsma, R. J. (1999). Movement reversals in ball catching. Experimental Brain Research, 129, 87-92. http://doi.org/10.1007/s002210050939

Morasso, P. (1981). Spatial control of arm movements. Experimental Brain Research. Experimentelle Hirnforschung. Expérimentation Cérébrale, 42(2), 223-7. http://doi.org/10.1007/BF00236911

Mottet, D., \& Bootsma, R. J. (1999). The dynamics of goal-directed rhythmical aiming. Biological Cybernetics, 245, 235-245.

Muceli, S., Falla, D., \& Farina, D. (2014). Reorganization of muscle synergies during multidirectional reaching in the horizontal plane with experimental muscle pain. Journal of Neurophysiology, 111(8), 1615-1630. http://doi.org/10.1152/jn.00147.2013

Newell, K. (1986). Constraint on the development of coordination. Motor Development in Children; Aspect of Coordination and Control, 341-360.

Newell, K. M., \& Vaillancourt, D. E. (2001). Dimensional change in motor learning. Human Movement Science, 20, 695-715. http://doi.org/S0167-9457(01)00073-2 [pii]

Peper, L., Bootsma, R. J., Mestre, D. R., \& Bakker, F. C. (1994). Catching Balls: How to Get the Hand to the Right Place at the Right Time. Journal of Experimental Psychology: Human Perception and Performance, 20(3), 591-612. 


\section{TASK CONSTRAINT ACT AT SYNERGIES AND END-EFFECTOR KINEMATICS}

Profeta, V. L. S., \& Turvey, M. T. (2018). Bernstein's levels of movement construction: A contemporary perspective. Human Movement Science, 57(November 2017), 111-133. http://doi.org/10.1016/j.humov.2017.11.013

Ranganathan, R., \& Newell, K. M. (2010). Goal and Execution Redundancy Levels Execution Redundancy Levels, (March 2012), 37-41.

Riley, M. a., Richardson, M. J., Shockley, K., \& Ramenzoni, V. C. (2011). Interpersonal $\begin{array}{lllll}\text { synergies. } & \text { Frontiers } & \text { in }\end{array}$ http://doi.org/10.3389/fpsyg.2011.00038

Riley, M. A., \& Turvey, M. T. (2002). Variability and Determinism iln Motor Behavior. Journal of Motor Behavior, 34(2), 37-41. http://doi.org/10.1080/00222890209601934

Romero, V., Kallen, R., Riley, M. A., \& Richardson, M. J. (2015). Can Discrete Joint Action Be Synergistic? Studying the Stabilization of Interpersonal Hand Coordination. $J$ Exp Psychol Hum Percept Perform, 41(5), 1223-1235. http://doi.org/10.1177/0963721412473755.Surging

Russo, M., D’Andola, M., Portone, A., Lacquaniti, F., \& d'Avella, A. (2014). Dimensionality of joint torques and muscle patterns for reaching. Frontiers in Computational Neuroscience, 8(MAR), 1-21. http://doi.org/10.3389/fncom.2014.00024

Saltzman, E., \& Scott Kelso, J. A. (1987). Skilled Actions: A Task-Dynamic Approach. Psychological Review, 94(1), 84-106. http://doi.org/10.1037/0033-295X.94.1.84

Schettino, L. F., Adamovich, S. V., \& Tunik, E. (2017). Coordination of pincer grasp and transport after mechanical perturbation of the index finger. Journal of Neurophysiology, 117(6), 2292-2297. http://doi.org/10.1152/jn.00642.2016

Schmidt, R. C., Carello, C., \& Turvey, M. T. (1990). Phase Transitions and Critical Fluctuations in the Visual Coordination of Rhythmic Movements Between People. Journal of Experimental Psychology: Human Perception and Performance, 16(2), 227-247. 


\section{TASK CONSTRAINT ACT AT SYNERGIES AND END-EFFECTOR KINEMATICS}

http://doi.org/10.1037/0096-1523.16.2.227

Scholz, J. P., \& Schöner, G. (1999). The uncontrolled manifold concept: Identifying control variables for a functional task. Experimental Brain Research, 126(3), 289-306. http://doi.org/10.1007/s002210050738

Schöner, G. (1995). Recent Developments and Problems in Human Movement Science and Their Conceptual Implications. Ecological Psychology, 7(4), 291-314. http://doi.org/10.1207/s15326969eco0704_5

Schöner, G. (2008). Motor equivalence and the uncontrolled manifold. 8th International Seminar on Speech Production 23, 23-28.

Sternad, D. (2018). It's not (only) the mean that matters: variability, noise and exploration in skill learning. Current Opinion in Behavioral Sciences, 20, 183-195. http://doi.org/10.1016/j.cobeha.2018.01.004

Tropea, P., Monaco, V., Coscia, M., Posteraro, F., \& Micera, S. (2013). Effects of early and intensive neuro-rehabilitative treatment on muscle synergies in acute post-stroke patients: A pilot study. Journal of NeuroEngineering and Rehabilitation, 10(1), 1-15. http://doi.org/10.1186/1743-0003-10-103

Tseng, Y.-W., Scholz, J. P., Schöner, G., \& Hotchkiss, L. (2003). Effect of accuracy constraint on joint coordination during pointing movements. Experimental Brain Research. Experimentelle Hirnforschung. Experimentation Cerebrale, 149, 276-288. http://doi.org/10.1007/s00221-002-1357-5

Turvey, M. T. (1990). Coordination. American Psychologist, 45(8), 938-953.

Turvey, M. T. (2007). Action and perception at the level of synergies. Human Movement Science, 26, 657-697. http://doi.org/10.1016/j.humov.2007.04.002

Turvey, M. T. ., Shaw, R. E. ., Reed, E. S. ., \& Mace, W. M. . (1981). Ecological laws of perceiving and acting: In reply to Fsdor and Pylyshyn (1981). Cognition, 9, 237-304. 


\section{TASK CONSTRAINT ACT AT SYNERGIES AND END-EFFECTOR KINEMATICS}

http://doi.org/10.1007/BF00730075

Valk, T. A., Mouton, L. J., \& Bongers, R. M. (2016). Joint-Angle Coordination Patterns Ensure Stabilization of a Body-Plus-Tool System in Point-to-Point Movements with a Rod. Frontiers in Psychology, 7(826), 826. http://doi.org/10.3389/fpsyg.2016.00826

Valk, T. A., Mouton, L. J., Otten, E., \& Bongers, R. M. (2019a). Fixed muscle synergies and their potential to improve the intuitive control of myoelectric assistive technology for upper extremities. Journal of NeuroEngineering and Rehabilitation, 16(1). http://doi.org/10.1186/s12984-018-0469-5

Valk, T. A., Mouton, L. J., Otten, E., \& Bongers, R. M. (2019b). Synergies reciprocally relate end-effector and joint-angles in rhythmic pointing movements. Scientific Reports, 9(1). http://doi.org/10.1038/s41598-019-53913-9

van Andel, C. J., Wolterbeek, N., Doorenbosch, C. A. M., Veeger, D. (H E. J. ), \& Harlaar, J. (2008). Complete 3D kinematics of upper extremity functional tasks. Gait and Posture, 27(1), 120-127. http://doi.org/10.1016/j.gaitpost.2007.03.002

Van De Kamp, C., \& Zaal, F. T. J. M. (2007). Prehension is really reaching and grasping. Experimental Brain Research, 182(1), 27-34. http://doi.org/10.1007/s00221-007-0968-2

Van Der Steen, M. C., \& Bongers, R. M. (2011). Joint angle variability and co-variation in a reaching with a rod task. Experimental Brain Research, 208, 411-422. http://doi.org/10.1007/s00221-010-2493-y

Verrel, J. (2010). Distributional properties and variance-stabilizing transformations for measures of uncontrolled manifold effects. Journal of Neuroscience Methods, 191(2), 166-170. http://doi.org/10.1016/j.jneumeth.2010.06.016

Wu, G., Van Der Helm, F. C. T., Veeger, H. E. J., Makhsous, M., Van Roy, P., Anglin, C., ... Buchholz, B. (2005). ISB recommendation on definitions of joint coordinate systems of various joints for the reporting of human joint motion - Part II: Shoulder, elbow, wrist and 
TASK CONSTRAINT ACT AT SYNERGIES AND END-EFFECTOR KINEMATICS

hand. Journal of Biomechanics, 38(5), 981-992. http://doi.org/10.1016/j.jbiomech.2004.05.042

Yang, J. F., \& Scholz, J. P. (2005). Learning a throwing task is associated with differential changes in the use of motor abundance. Experimental Brain Research, 163, 137-158. http://doi.org/10.1007/s00221-004-2149-x

Yang, J. F., Scholz, J. P., \& Latash, M. L. (2007). The role of kinematic redundancy in adaptation of reaching. Experimental Brain Research, 176, 54-69. http://doi.org/10.1007/s00221-006-0602-8

Zaal, F. T. J. M., \& Bongers, R. M. (2014). Movements of Individual Digits in Bimanual Prehension Are Coupled into a Grasping Component, 9(5), 1-6. http://doi.org/10.1371/journal.pone.0097790

Footnote

${ }^{1}$ Note that the outcomes of the UCM analysis are similar if all missed interception trials are included in the analysis.

Acknowledgements

We thank Wim Kaan for his help in creating the task display, Ester Loeve and Jasper Tonkelaar for their help collecting the data, and Tim Valk for the fruitful discussions while writing this paper. The project leading to this publication has received funding from Excellence Initiative of Aix-Marseille University - A*MIDEX, a French "Investissements d'Avenir" program. 